\title{
Iterated commutators of multilinear Calderón-Zygmund maximal operators on some function spaces
}

Zengyan $\mathrm{Si}^{1 *}$ (D) and Pu Zhang ${ }^{2}$

\section{"Correspondence:}

zengyan@hpu.edu.cn

'School of Mathematics and Information Science, Henan Polytechnic University, Jiaozuo, People's Republic of China

Full list of author information is available at the end of the article

\begin{abstract}
Let $T^{*}$ be a multilinear Calderón-Zygmund maximal operator. In this paper, we study iterated commutators of $T^{*}$ and pointwise multiplication with functions in Lipschitz spaces. More precisely, we give some new estimates for this kind of commutators under some Dini-type conditions on Lebesgue spaces, homogenous Lipschitz spaces, and homogenous Triebel-Lizorkin spaces.
\end{abstract}

MSC: 42B25; 47G10

Keywords: Maximal multilinear operators; Calderón-Zygmund operator; Commutators

\section{Introduction and main results}

For any $a>0$, we say that $\omega \in \operatorname{Dini}(a)$ if

$$
|\omega|_{\operatorname{Dini}(a)}=\int_{0}^{1} \frac{\omega^{a}(t)}{t} d t<\infty
$$

where $\omega(t):[0, \infty) \mapsto[0, \infty)$ is a nondecreasing function with $0<\omega(1)<\infty$.

We say that $T$ is a multilinear Calderón-Zygmund operator with kernel of type $\omega(t)$, denoted by $m$-linear $\omega$-CZO, if $T$ can be extended to a bounded multilinear operator from $L^{q_{1}}\left(\mathbb{R}^{n}\right) \times \cdots \times L^{q_{m}}\left(\mathbb{R}^{n}\right)$ to $L^{q, \infty}\left(\mathbb{R}^{n}\right)$ for some $1<q, q_{1}, \ldots, q_{m}<\infty$ with $\frac{1}{q_{1}}+\cdots+\frac{1}{q_{m}}=\frac{1}{q}$, or from $L^{q_{1}}\left(\mathbb{R}^{n}\right) \times \cdots \times L^{q_{m}}\left(\mathbb{R}^{n}\right)$ to $L^{1}\left(\mathbb{R}^{n}\right)$ for some $1<q_{1}, \ldots, q_{m}<\infty$ with $\frac{1}{q_{1}}+\cdots+\frac{1}{q_{m}}=$ 1 , and if there exists a function $K$ defined off the diagonal $x=y_{1}=\cdots=y_{m}$ in $\left(\mathbb{R}^{n}\right)^{m+1}$, satisfying

$$
\overrightarrow{T f}(x)=T\left(f_{1}, \ldots, f_{m}\right)(x)=\int_{\left(\mathbb{R}^{n}\right)^{m}} K\left(x, y_{1}, \ldots, y_{m}\right) f_{1}\left(y_{1}\right) \cdots f_{m}\left(y_{m}\right) d y_{1} \cdots d y_{m}
$$

for all $x \notin \bigcap_{j=1}^{m} \operatorname{supp} f_{j}$ and $f_{j} \in C_{c}^{\infty}\left(\mathbb{R}^{n}\right), j=1, \ldots, m$, and if there exists a constant $A>0$ such that

$$
\left|K\left(x, y_{1}, \ldots, y_{m}\right)\right| \leq \frac{A}{\left(\left|x-y_{1}\right|+\cdots+\left|x-y_{m}\right|\right)^{m n}}
$$

(c) The Author(s) 2019. This article is distributed under the terms of the Creative Commons Attribution 4.0 International License (http://creativecommons.org/licenses/by/4.0/), which permits unrestricted use, distribution, and reproduction in any medium, provided you give appropriate credit to the original author(s) and the source, provide a link to the Creative Commons license, and indicate if changes were made. 
for all $\left(x, y_{1}, \ldots, y_{m}\right) \in\left(\mathbb{R}^{n}\right)^{m+1}$ with $x \neq y_{j}$ for some $j \in\{1,2, \ldots, m\}$, and

$$
\begin{aligned}
& \left|K\left(x, y_{1}, \ldots, y_{m}\right)-K\left(x^{\prime}, y_{1}, \ldots, y_{m}\right)\right| \\
& \quad \leq \frac{A}{\left(\left|x-y_{1}\right|+\cdots+\left|x-y_{m}\right|\right)^{m n}} \omega\left(\frac{\left|x-x^{\prime}\right|}{\left|x-y_{1}\right|+\cdots+\left|x-y_{m}\right|}\right)
\end{aligned}
$$

whenever $\left|x-x^{\prime}\right| \leq \frac{1}{m+1} \max _{1 \leq j \leq m}\left|x-y_{j}\right|$, and

$$
\begin{aligned}
& \left|K\left(x, y_{1}, \ldots, y_{j}, \ldots, y_{m}\right)-K\left(x, y_{1}, \ldots, y_{j}^{\prime}, \ldots, y_{m}\right)\right| \\
& \quad \leq \frac{A}{\left(\left|x-y_{1}\right|+\cdots+\left|x-y_{m}\right|\right)^{m n}} \omega\left(\frac{\left|y_{j}-y_{j}^{\prime}\right|}{\left|x-y_{1}\right|+\cdots+\left|x-y_{m}\right|}\right)
\end{aligned}
$$

whenever $\left|y_{j}-y_{j}^{\prime}\right| \leq \frac{1}{m+1} \max _{1 \leq j \leq m}\left|x-y_{j}\right|$.

When $\omega(x)=x^{\gamma}$ for some $\gamma>0$, the $m$-linear $\omega$-CZO is exactly the multilinear Calderón-Zygmund operator studied by Grafakos and Torres in [11]. The multilinear Calderón-Zygmund operators were introduced and first studied by Coifman and Meyer [5-7] and later by Grafakos and Torres [11, 12]. The study of such operators has attracted the interest of many experts; see, for example, $[4,14,24]$ and the reference therein. Recently, many mathematicians are concerned to remove or replace the smoothness condition on the kernels; see, for example $[1,8-10,13,15,21]$. In this paper, we mainly investigate the maximal operator and give some new estimates for its iterated commutators on some function spaces.

The maximal truncated operator $T^{*}$ is defined by

$$
T^{*}(\vec{f})(x)=\sup _{\delta>0}\left|T_{\delta}\left(f_{1}, \ldots, f_{m}\right)(x)\right|
$$

where $T_{\delta}$ are the smooth truncations of $T$, that is,

$$
T_{\delta}\left(f_{1}, \ldots, f_{m}\right)(x)=\int_{\left|x-y_{1}\right|^{2}+\cdots+\left|x-y_{m}\right|^{2}>\delta^{2}} K\left(x, y_{1}, \ldots, y_{m}\right) f_{1}\left(y_{1}\right) \cdots f_{m}\left(y_{m}\right) d y_{1} \cdots d y_{m}
$$

For the maximal truncated operator $T^{*}$ and a collection of locally integrable functions $\vec{b}=\left(b_{1}, \ldots, b_{m}\right)$, we define the iterated commutator $T_{\Pi \vec{b}}^{*}$ by

$$
\begin{aligned}
T_{\Pi \vec{b}}^{*}(\vec{f})(x) & =\sup _{\delta>0}\left|\left[b_{1},\left[b_{2}, \ldots\left[b_{m-1},\left[b_{m}, T_{\delta}\right]_{m}\right]_{m-1} \ldots\right]_{2}\right]_{1}(\vec{f})(x)\right| \\
& =\sup _{\delta>0}\left|\int_{\left|x-y_{1}\right|^{2}+\cdots+\left|x-y_{m}\right|^{2}>\delta^{2}} \prod_{j=1}^{m}\left(b_{j}(x)-b_{j}\left(y_{j}\right)\right) K\left(x, y_{1}, \ldots, y_{m}\right) \prod_{i=1}^{m} f_{i}\left(y_{i}\right) d \vec{y}\right| .
\end{aligned}
$$

The iterated commutators of multilinear singular integral operators with BMO functions have been studied by a large number of people; see, for example, $[2,18,19]$. On the other hand, commutators of multilinear singular integral operators with Lipschitz functions have been the subject of many recent papers. In 1995, Paluszyński [17] proved that the commutator generated by Calderón-Zygmund operators with classical kernel and Lipschitz functions is bounded from the Lebesgue space to the Lebesgue space and to the homogenous Triebel-Lizorkin space. The multilinear analogues of the results in [17] 
were given by Wang and $\mathrm{Xu}$ [23] and by Mo and Lu [16]. Finally, Sun and Zhang [22] relaxed the smooth condition assumed on the kernel to Dini-type condition. It is natural to ask whether, under the Dini-type condition, the iterated commutators of multilinear Calderón-Zygmund maximal operators and pointwise multiplication with functions in Lipschitz space share similar boundedness properties? In this paper, we give a positive answer. The main result reads as follows.

Theorem 1.1 Suppose $\omega \in \operatorname{Dini}(1)$ and $b_{j} \in \operatorname{Lip}_{\beta_{j}}$ with $0<\beta_{j}<1$ for $j=1, \ldots, m$ and $\beta=$ $\beta_{1}+\cdots+\beta_{m}$. If $1<p_{1}, \ldots, p_{m}<\infty, 0<q<\infty$, and $1 / p_{j}>\beta_{j} / n$ with $1 / q=1 / p_{1}+\cdots+1 / p_{m}-$ $\beta / n$, then

$$
\left\|T_{\Pi \vec{b}}^{*} \vec{f}\right\|_{L^{q}} \lesssim \prod_{i=1}^{m}\left\|b_{i}\right\|_{\operatorname{Lip}_{\beta_{i}}} \prod_{i=1}^{m}\left\|f_{i}\right\|_{L^{p_{i}}}
$$

Theorem 1.2 Suppose $b_{j} \in \operatorname{Lip}_{\beta_{j}}$ with $0<\beta_{j}<1$ for $j=1, \ldots$, m and $\beta=\beta_{1}+\cdots+\beta_{m}$. If $1<p_{1}, \ldots, p_{m}<\infty, 0<1 / p_{j}<\beta_{j} / n, 0<\beta-n / p<1$ with $1 / p=1 / p_{1}+\cdots+1 / p_{m}$, and $\omega$ satisfies

$$
\int_{0}^{1} \frac{\omega(t)}{t^{1+\beta-n / p}} d t<\infty
$$

then

$$
\left\|T_{\Pi \vec{b}}^{*} \vec{f}\right\|_{\operatorname{Lip}_{\beta-n / p}} \lesssim \prod_{i=1}^{m}\left\|b_{i}\right\|_{\operatorname{Lip}_{\beta_{i}}} \prod_{i=1}^{m}\left\|f_{i}\right\|_{L^{p_{i}}} .
$$

Theorem 1.3 Suppose $b_{j} \in \operatorname{Lip}_{\beta_{j}}$ with $0<\beta_{j}<1$ for $j=1, \ldots, m$ and $\beta=\beta_{1}+\cdots+\beta_{m}$. If $1<p_{1}, \ldots, p_{m}<\infty$ with $1 / p=1 / p_{1}+\cdots+1 / p_{m}$ and $\omega$ satisfies

$$
\int_{0}^{1} \frac{\omega(t)}{t^{1+\beta}} d t<\infty
$$

then

$$
\left\|T_{\Pi \vec{b}}^{*} \vec{f}\right\|_{\dot{F}_{p}^{\beta, \infty}} \lesssim \prod_{i=1}^{m}\left\|b_{i}\right\|_{\operatorname{Lip}_{\beta_{i}}} \prod_{i=1}^{m}\left\|f_{i}\right\|_{L^{p_{i}}} .
$$

In the next section, we give some definitions and preliminaries. We focus on the proof of Theorem 1.1 in Sect. 3. The proof of Theorems 1.2 and 1.3 is given in Sect. 4. The notation $A \lesssim B$ stands for $A \leq C B$ for some positive constant $C$ independent of $A$ and $B$.

\section{Preliminaries}

Definition 2.1 Given a locally integrable function $f$, define the fractional maximal function by

$$
M_{\beta, r} f(x)=\sup _{x \in Q}\left(\frac{1}{|Q|^{1-\beta r / n}} \int_{Q}|f(y)|^{r} d y\right)^{\frac{1}{r}}, \quad r \geq 1,
$$


when $0 \leq \beta<n / r$. If $\beta=0$ and $r=1$, then $M_{0,1} f=M f$ denotes the usual Hardy-Littlewood maximal function. For $\delta>0$, we denote $M_{\delta}$ by $M_{\delta} f=M\left(|f|^{\delta}\right)^{\frac{1}{\delta}}$.

The sharp maximal function $M^{\sharp}$ is given by

$$
M^{\sharp} f(x)=\sup _{Q \ni x} \inf _{C} \frac{1}{|Q|} \int_{Q}|f(y)-C| d y \approx \sup _{Q \ni x} \frac{1}{|Q|} \int_{Q}\left|f(y)-f_{Q}\right| d y,
$$

where $f_{Q}$ denotes the average of $f$ over cube $Q$, and we denote $M_{\delta}^{\sharp}$ by $M_{\delta}^{\sharp} f(x)=M^{\sharp}\left(|f|^{\delta}\right)^{\frac{1}{\delta}}(x)$.

Definition 2.2 ([17]) For $\beta>0$, the homogenous Lipschitz space $\operatorname{Lip}_{\beta}\left(\mathbb{R}^{n}\right)$ is the space of functions $f$ such that

$$
\|f\|_{\operatorname{Lip}_{\beta}\left(\mathbb{R}^{n}\right)}=\sup _{x, h \in \mathbb{R}^{n}, h \neq 0} \frac{\left|\Delta_{h}^{[\beta]+1} f(x)\right|}{|h|^{\beta}}<\infty
$$

where $\Delta_{h}^{k}$ denotes the $k$ th difference operator.

To prove Theorems 1.1, 1.2, and 1.3, we need the following lemmas.

Lemma 2.1 ([17]) Let $b \in \operatorname{Lip}_{\beta}\left(\mathbb{R}^{n}\right), 0<\beta<1$. For any cubes $Q^{\prime}, Q$ in $\mathbb{R}^{n}$ such that $Q^{\prime} \subset Q$, we have

$$
\left|b_{Q^{\prime}}-b_{Q}\right| \lesssim\|b\|_{\operatorname{Lip}_{\beta}\left(\mathbb{R}^{n}\right)}|Q|^{\beta / n}
$$

Lemma $2.2([17])$

(1) For $0<\beta<1$ and $1 \leq q<\infty$, we have

$$
\|f\|_{\operatorname{Lip}_{\beta}\left(\mathbb{R}^{n}\right)} \approx \sup _{Q} \frac{1}{|Q|^{1+n / \beta}} \int_{Q}\left|f-f_{Q}\right| \approx \sup _{Q} \frac{1}{|Q|^{n / \beta}}\left(\int_{Q}\left|f-f_{Q}\right|^{q}\right)^{\frac{1}{q}} .
$$

(2) For $0<\beta<1$ and $1 \leq p<\infty$, we have

$$
\|f\|_{\dot{F}_{p}^{\beta, \infty}} \approx\left\|\sup _{Q} \frac{1}{|Q|^{1+n / \beta}} \int_{Q}\left|f-f_{Q}\right|\right\|_{L^{p}}
$$

Lemma 2.3 ([20]) Let $\frac{1}{p}=\frac{1}{p_{1}}+\cdots+\frac{1}{p_{m}}$ and $\vec{\omega} \in A_{\vec{p}}$. Let $T$ be an m-linear $\omega$-CZO with $\omega \in \operatorname{Dini}(1)$.

(1) If $1<p_{1}, \ldots, p_{m}<\infty$, then

$$
\left\|T^{*} \vec{f}\right\|_{L^{p}\left(v_{\vec{\omega}}\right)} \lesssim \prod_{i=1}^{m}\left\|f_{i}\right\|_{L^{p_{i}}\left(\omega_{i}\right)}
$$

(2) If $1 \leq p_{1}, \ldots, p_{m}<\infty$, then

$$
\left\|T^{*} \vec{f}\right\|_{L^{p, \infty}\left(\nu_{\vec{\omega}}\right)} \lesssim \prod_{i=1}^{m}\left\|f_{i}\right\|_{L^{p_{i}\left(\omega_{i}\right)}} .
$$




\section{Proof of Theorem 1.1}

We borrow some ideas from [19]. Since the proof of Theorem 1.1 follows from similar steps in [22], we omit the proof. We just give three key lemmas.

Let $u, v \in C^{\infty}([0, \infty))$ be such that $\left|u^{\prime}(t)\right| \leq C t^{-1},\left|v^{\prime}(t)\right| \leq C t^{-1}$, and

$$
\chi_{[2, \infty)}(t) \leq u(t) \leq \chi_{[1, \infty)}(t), \quad \chi_{[1,2]}(t) \leq v(t) \leq \chi_{[1 / 2,3]}(t) .
$$

For simplicity, we denote

$$
\begin{aligned}
& K_{u, \eta}\left(x, y_{1}, \ldots, y_{m}\right)=K\left(x, y_{1}, \ldots, y_{m}\right) u\left(\frac{\left|x-y_{1}\right|+\cdots+\left|x-y_{m}\right|}{\eta}\right), \\
& K_{v, \eta}\left(x, y_{1}, \ldots, y_{m}\right)=K\left(x, y_{1}, \ldots, y_{m}\right) v\left(\frac{\left|x-y_{1}\right|+\cdots+\left|x-y_{m}\right|}{\eta}\right),
\end{aligned}
$$

and

$$
\begin{aligned}
& U_{\eta}(\vec{f})(x)=\int_{\left(\mathbb{R}^{n}\right)^{m}} K_{u, \eta}\left(x, y_{1}, \ldots, y_{m}\right) \prod_{i=1}^{m} f_{i}\left(y_{i}\right) d y_{1} \cdots d y_{m}, \\
& V_{\eta}(\vec{f})(x)=\int_{\left(\mathbb{R}^{n}\right)^{m}} K_{v, \eta}\left(x, y_{1}, \ldots, y_{m}\right) \prod_{i=1}^{m} f_{i}\left(y_{i}\right) d y_{1} \cdots d y_{m} .
\end{aligned}
$$

Then we define the maximal operators

$$
U^{*}(\vec{f})(x)=\sup _{\eta>0}\left|U_{\eta}(\vec{f})(x)\right| \quad \text { and } \quad V^{*}(\vec{f})(x)=\sup _{\eta>0}\left|V_{\eta}(\vec{f})(x)\right|
$$

It is easy to get $T^{*}(\vec{f}) \leq U^{*}(\vec{f})(x)+V^{*}(\vec{f})(x)$. Next, we show that the functions $K_{u, \eta}$ and $K_{v, \eta}$ satisfy some smoothness properties.

Lemma 3.1 For any $j=0,1,2, \ldots, m$, we have

$$
\begin{aligned}
& \left|K_{u, \eta}\left(y_{0}, \ldots, y_{j}, \ldots, y_{m}\right)-K_{u, \eta}\left(y_{0}, \ldots, y_{j}^{\prime}, \ldots, y_{m}\right)\right| \\
& \quad \lesssim \frac{\omega\left(\frac{\left|y_{j}-y_{j}^{\prime}\right|}{\left|y_{0}-y_{1}\right|+\cdots+\left|y_{0}-y_{m}\right|}\right)}{\left(\left|y_{0}-y_{1}\right|+\cdots+\left|y_{0}-y_{m}\right|\right)^{m n}}+\frac{\left|y_{j}-y_{j}^{\prime}\right|}{\left(\left|y_{0}-y_{1}\right|+\cdots+\left|y_{0}-y_{m}\right|\right)^{m n+1}}
\end{aligned}
$$

and

$$
\begin{aligned}
& \left|K_{v, \eta}\left(y_{0}, \ldots, y_{j}, \ldots, y_{m}\right)-K_{v, \eta}\left(y_{0}, \ldots, y_{j}^{\prime}, \ldots, y_{m}\right)\right| \\
& \quad \lesssim \frac{\omega\left(\frac{\left|y_{j}-y_{j}^{\prime}\right|}{\left|y_{0}-y_{1}\right|+\cdots+\left|y_{0}-y_{m}\right|}\right)}{\left(\left|y_{0}-y_{1}\right|+\cdots+\left|y_{0}-y_{m}\right|\right)^{m n}}+\frac{\left|y_{j}-y_{j}^{\prime}\right|}{\left(\left|y_{0}-y_{1}\right|+\cdots+\left|y_{0}-y_{m}\right|\right)^{m n+1}}
\end{aligned}
$$

whenever $\left|y_{j}-y_{j}^{\prime}\right| \leq \frac{1}{m+1} \max _{0 \leq j \leq m}\left|y_{0}-y_{j}\right|$. 
Proof We just give the estimate for $K_{u, \eta}$, since $K_{v, \eta}$ can be estimated in a similar way with slight modifications. Without loss of generality, assuming that $j=0$, we estimate

$$
\begin{aligned}
\left|K_{u, \eta}\left(y_{0}, y_{1}, \ldots, y_{m}\right)-K_{u, \eta}\left(y_{0}^{\prime}, y_{1}, \ldots, y_{m}\right)\right| & \mid K\left(y_{0}, y_{1}, \ldots, y_{m}\right) u\left(\frac{\left|y_{0}-y_{1}\right|+\cdots+\left|y_{0}-y_{m}\right|}{\eta}\right) \\
& \quad-K\left(y_{0}^{\prime}, y_{1}, \ldots, y_{m}\right) u\left(\frac{\left|y_{0}^{\prime}-y_{1}\right|+\cdots+\left|y_{0}^{\prime}-y_{m}\right|}{\eta}\right) \mid \\
= & \mid\left[K\left(y_{0}, y_{1}, \ldots, y_{m}\right)-K\left(y_{0}^{\prime}, y_{1}, \ldots, y_{m}\right)\right] u\left(\frac{\left|y_{0}^{\prime}-y_{1}\right|+\cdots+\left|y_{0}^{\prime}-y_{m}\right|}{\eta}\right) \\
& -K\left(y_{0}, y_{1}, \ldots, y_{m}\right) \\
& \times\left[u\left(\frac{\left|y_{0}^{\prime}-y_{1}\right|+\cdots+\left|y_{0}^{\prime}-y_{m}\right|}{\eta}\right)-u\left(\frac{\left|y_{0}-y_{1}\right|+\cdots+\left|y_{0}-y_{m}\right|}{\eta}\right)\right] \mid \\
\lesssim & \left|K\left(y_{0}, y_{1}, \ldots, y_{m}\right)-K\left(y_{0}^{\prime}, y_{1}, \ldots, y_{m}\right)\right| \\
& +\mid K\left(y_{0}, y_{1}, \ldots, y_{m}\right) \\
& \quad\left[u\left(\frac{\left|y_{0}^{\prime}-y_{1}\right|+\cdots+\left|y_{0}^{\prime}-y_{m}\right|}{\eta}\right)-u\left(\frac{\left|y_{0}-y_{1}\right|+\cdots+\left|y_{0}-y_{m}\right|}{\eta}\right)\right] \mid \\
\doteq I+I I . &
\end{aligned}
$$

Since $\left|y_{0}-y_{0}^{\prime}\right| \leq \frac{1}{m+1} \max _{0 \leq j \leq m}\left|y_{0}-y_{j}\right|$, by (1.3) we have

$$
I \lesssim \frac{1}{\left(\left|y_{0}-y_{1}\right|+\cdots+\left|y_{0}-y_{m}\right|\right)^{m n}} \omega\left(\frac{\left|y_{0}-y_{0}^{\prime}\right|}{\left|y_{0}-y_{1}\right|+\cdots+\left|y_{0}-y_{m}\right|}\right)
$$

It remains to estimate $I I$. By the mean value theorem there is $t_{0}$ between $\frac{\left|y_{0}^{\prime}-y_{1}\right|+\cdots+\left|y_{0}^{\prime}-y_{m}\right|}{\eta}$ and $\frac{\left|y_{0}-y_{1}\right|+\cdots+\left|y_{0}-y_{m}\right|}{\eta}$ such that

$$
\begin{aligned}
& \left|u\left(\frac{\left|y_{0}^{\prime}-y_{1}\right|+\cdots+\left|y_{0}^{\prime}-y_{m}\right|}{\eta}\right)-u\left(\frac{\left|y_{0}-y_{1}\right|+\cdots+\left|y_{0}-y_{m}\right|}{\eta}\right)\right| \\
& \quad=\left|u^{\prime}\left(t_{0}\right)\right| \frac{\left|y_{0}^{\prime}-y_{1}\right|+\cdots+\left|y_{0}^{\prime}-y_{m}\right|}{\eta}-\frac{\left|y_{0}-y_{1}\right|+\cdots+\left|y_{0}-y_{m}\right|}{\eta} \mid \\
& \quad \leq \frac{1}{t_{0}} \frac{|| y_{0}^{\prime}-y_{1}|-| y_{0}-y_{1}||+\cdots+|| y_{0}^{\prime}-y_{m}|-| y_{0}-y_{m}||}{\eta} \\
& \quad \leq \frac{1}{t_{0}} \frac{m\left|y_{0}-y_{0}^{\prime}\right|}{\eta} .
\end{aligned}
$$

Again, since $\left|y_{0}-y_{0}^{\prime}\right| \lesssim \frac{1}{m+1} \max _{0 \leq j \leq m}\left|y_{0}-y_{j}\right|$, we have

$$
\begin{aligned}
\left|y_{0}^{\prime}-y_{1}\right|+\cdots+\left|y_{0}^{\prime}-y_{m}\right| & =\left|y_{0}-y_{1}+y_{0}^{\prime}-y_{0}\right|+\cdots+\left|y_{0}-y_{m}+y_{0}^{\prime}-y_{0}\right| \\
& \geq\left|y_{0}-y_{1}\right|+\cdots+\left|y_{0}-y_{m}\right|-m\left|y_{0}-y_{0}^{\prime}\right|
\end{aligned}
$$




$$
\begin{aligned}
& \geq\left|y_{0}-y_{1}\right|+\cdots+\left|y_{0}-y_{m}\right|-\frac{m}{m+1} \max _{0 \leq j \leq m}\left|y_{0}-y_{0}^{\prime}\right| \\
& \geq \frac{\left|y_{0}-y_{1}\right|+\cdots+\left|y_{0}-y_{m}\right|}{m+1} .
\end{aligned}
$$

From this,

$$
\begin{aligned}
\frac{1}{t_{0}} & \lesssim \max \left\{\frac{\eta}{\left|y_{0}^{\prime}-y_{1}\right|+\cdots+\left|y_{0}^{\prime}-y_{m}\right|}, \frac{\eta}{\left|y_{0}-y_{1}\right|+\cdots+\left|y_{0}-y_{m}\right|}\right\} \\
& \lesssim \frac{\eta}{\left|y_{0}-y_{1}\right|+\cdots+\left|y_{0}-y_{m}\right|},
\end{aligned}
$$

and therefore

$$
\begin{aligned}
& \left|u\left(\frac{\left|y_{0}^{\prime}-y_{1}\right|+\cdots+\left|y_{0}^{\prime}-y_{m}\right|}{\eta}\right)-u\left(\frac{\left|y_{0}-y_{1}\right|+\cdots+\left|y_{0}-y_{m}\right|}{\eta}\right)\right| \\
& \quad \lesssim \frac{\left|y_{0}-y_{0}^{\prime}\right|}{\left|y_{0}-y_{1}\right|+\cdots+\left|y_{0}-y_{m}\right|} .
\end{aligned}
$$

This, together with the size condition (1.2), implies that

$$
I I \lesssim \frac{\left|y_{0}-y_{0}^{\prime}\right|}{\left(\left|y_{0}-y_{1}\right|+\cdots+\left|y_{0}-y_{m}\right|\right)^{m n+1}} .
$$

This ends the proof of Lemma 3.1.

Lemma 3.2 Let $\frac{1}{p}=\frac{1}{p_{1}}+\cdots+\frac{1}{p_{2}}$ and $\vec{\omega} \in A_{\vec{p}}$. Then we have:

(1) If $1<p_{1}, \ldots, p_{m}<\infty$, then

$$
\left\|U^{*} \vec{f}\right\|_{L^{p}\left(v_{\vec{\omega}}\right)} \lesssim \prod_{i=1}^{m}\left\|f_{i}\right\|_{L^{p_{i}\left(\omega_{i}\right)} .}
$$

(2) If $1 \leq p_{1}, \ldots, p_{m}<\infty$, then

$$
\left\|U^{*} \vec{f}\right\|_{L^{p, \infty}\left(v_{\bar{\omega}}\right)} \lesssim \prod_{i=1}^{m}\left\|f_{i}\right\|_{L^{p_{i}\left(\omega_{i}\right)}} .
$$

Similar estimates hold for $V^{*}$.

Proof Lemma 3.2 is a consequence of Lemma 2.3, Lemma 3.1, and Theorem 1.3 in [3].

For the maximal truncated operator $T^{*}$ and a collection of locally integrable functions $\vec{b}=\left(b_{1}, \ldots, b_{m}\right)$, we define the commutator $T_{\Sigma \vec{b}}^{*}$ by

$$
T_{\Sigma \vec{b}}^{*}\left(f_{1}, \ldots, f_{m}\right)=\sum_{j=1}^{m} T_{\vec{b}}^{* j}(\vec{f}),
$$

where

$$
T_{\vec{b}}^{* j}(\vec{f})(x)=\left[b_{j}, T^{*}\right]_{j}(\vec{f})(x)=\sup _{\delta>0}\left|b_{j}(x) T_{\delta}\left(f_{1}, \ldots, f_{m}\right)(x)-T_{\delta}\left(f_{1}, \ldots, b_{j} f_{j}, \ldots, f_{m}\right)(x)\right| .
$$


Next, we give the key lemma, which plays important role in the proof of Theorem 1.1. We just consider the case $m=2$ for simplicity.

Lemma 3.3 Let $T$ be an m-linear $\omega$-CZO with $\omega \in \operatorname{Dini}(1)$. Then we have:

(i) If $b_{1} \in \operatorname{Lip}_{\beta_{1}}$ and $b_{2} \in \operatorname{Lip}_{\beta_{2}}$ with $0<\beta_{1}, \beta_{2}<1,0<\delta<\epsilon<1 / 2$, then

$$
\begin{aligned}
& M_{\delta}^{\sharp} T_{\Pi \vec{b}}^{*}\left(f_{1}, f_{2}\right)(x) \\
& \lesssim\left\{\prod_{i=1}^{2}\left\|b_{i}\right\|_{\operatorname{Lip}_{\beta_{i}}} M_{\epsilon, \beta}\left(T^{*}\left(f_{1}, f_{2}\right)\right)(x)+\left\|b_{1}\right\|_{\operatorname{Lip}_{\beta_{1}}} M_{\epsilon, \beta_{1}}\left(T_{\vec{b}}^{* 2}\left(f_{1}, f_{2}\right)\right)(x)\right. \\
& \quad+\left\|b_{2}\right\|_{\operatorname{Lip}_{\beta_{1}}} M_{\epsilon, \beta_{2}}\left(T_{\vec{b}}^{* 1}\left(f_{1}, f_{2}\right)\right)(x) \\
& \left.\quad+\prod_{i=1}^{2}\left\|b_{i}\right\|_{\operatorname{Lip}_{\beta_{i}}} M_{1, \beta_{1}}\left(f_{1}\right)(x) M_{1, \beta_{2}}\left(f_{2}\right)(x)\right\} .
\end{aligned}
$$

(ii) Suppose that $b_{j} \in \operatorname{Lip}_{\beta}, j=1,2,0<\beta<1$, and $0<\delta<\epsilon<1 / 2<1<n / \beta$. Then

$$
\begin{aligned}
M_{\delta}^{\sharp} & T_{\Sigma \vec{b}}^{*}\left(f_{1}, f_{2}\right)(x) \\
\lesssim & \|b\|_{\operatorname{Lip}_{\beta}}\left\{M_{\epsilon, \beta}\left(T^{*}\left(f_{1}, f_{2}\right)\right)(x)+M_{1, \beta}\left(f_{1}\right)(x) M\left(f_{2}\right)(x)\right. \\
& \left.+M_{1, \beta}\left(f_{2}\right)(x) M\left(f_{1}\right)(x)\right\} .
\end{aligned}
$$

Proof (i) We need two auxiliary maximal operators. The key role in the proof is played by the maximal operators $U_{\Pi b}^{*}$ and $V_{\Pi b}^{*}$ defined by

$$
\begin{aligned}
\begin{aligned}
U_{\Pi b}^{*}(\vec{f})(x) & =\sup _{\eta>0}\left|\left[b_{1},\left[b_{2}, U_{\eta}\right]_{2}\right]_{1}(\vec{f})(x)\right| \\
& =\sup _{\eta>0}\left|\int_{\left(\mathbb{R}^{n}\right)^{m}} K_{u, \eta}\left(x, y_{1}, y_{2}\right) \prod_{j=1}^{2}\left(b_{j}(x)-b_{j}\left(y_{j}\right)\right) \prod_{i=1}^{2} f_{i}\left(y_{i}\right) d y_{1} d y_{2}\right|, \\
\left.V_{\Pi b}^{*}(\vec{f})(x)\right) & =\sup _{\eta>0}\left|\left[b_{1},\left[b_{2}, V_{\eta}\right]_{2}\right]_{1}(\vec{f})(x)\right| \\
& \left.=\sup _{\eta>0} \mid \int_{\left(\mathbb{R}^{n}\right)^{2}} K_{v, \eta}\left(x, y_{1}, y_{2}\right) \prod_{j=1}^{2} b_{j}(x)-b_{j}\left(y_{j}\right)\right) \prod_{i=1}^{2} f_{i}\left(y_{i}\right) d y_{1} d y_{2} \mid .
\end{aligned}
\end{aligned}
$$

It is easy to get that $T_{\Pi b}^{*}(\vec{f}) \leq U_{\Pi b}^{*}(\vec{f})(x)+V_{\Pi b}^{*}(\vec{f})(x)$. We need to prove (3.1) for $U_{\Pi \vec{b}}^{*}$ and $V_{\Pi \vec{b}}^{*}$. We just give the proof for $U_{\Pi \vec{b}}^{*}$, since the proof for $V_{\Pi \vec{b}}^{*}$ is almost the same. Fix $x \in \mathbb{R}^{n}$ and denote by $Q=Q\left(x_{Q}, l\right)$ the cube centered at $x_{Q}$ and containing $x$ with side length $l$. Denote $c=\sup _{\eta>0}\left|c_{\eta}\right|$ and $\lambda_{i}=\left(b_{i}\right)_{Q^{*}}=\frac{1}{\left|Q^{*}\right|} \int_{Q^{*}} b_{i}(y) d y$, where $Q^{*}=8 \sqrt{n} Q$. For any $z \in \mathbb{R}^{n}$, we have

$$
\begin{aligned}
\left|U_{\Pi \vec{b}}^{*}\left(f_{1}, f_{2}\right)(z)-c\right| \leq & \left|\left(b_{1}(z)-\lambda_{1}\right)\left(b_{2}(z)-\lambda_{2}\right) U^{*}\left(f_{1}, f_{2}\right)(z)\right| \\
& +\sup _{\eta}\left|\left(b_{1}(z)-\lambda_{1}\right)\left[b_{2}, U_{\eta}\right]_{2}\left(f_{1}, f_{2}\right)(z)\right|
\end{aligned}
$$




$$
\begin{aligned}
& +\sup _{\eta}\left|\left(b_{2}(z)-\lambda_{2}\right)\left[b_{1}, U_{\eta}\right]_{1}\left(f_{1}, f_{2}\right)(z)\right| \\
& +\left|U^{*}\left(\left(b_{1}-\lambda_{1}\right) f_{1},\left(b_{2}-\lambda_{2}\right) f_{2}\right)(z)-\sup _{\eta>0}\right| c_{\eta}|| .
\end{aligned}
$$

Thus we have

$$
\begin{aligned}
\left(\left.\frac{1}{|Q|} \int_{Q}|| U_{\Pi \vec{b}}^{*}\left(f_{1}, f_{2}\right)(z)\right|^{\delta}-|c|^{\delta} \mid d z\right)^{\frac{1}{\delta}} & \\
\leq & \left(\frac{1}{|Q|} \int_{Q}\left|U_{\Pi \vec{b}}^{*}\left(f_{1}, f_{2}\right)(z)-\sup _{\eta>0}\right| c_{\eta}||^{\delta} d z\right)^{\frac{1}{\delta}} \\
\leq & \left(\frac{1}{|Q|} \int_{Q}\left|\left(b_{1}(z)-\lambda_{1}\right)\left(b_{2}(z)-\lambda_{2}\right) U^{*}\left(f_{1}, f_{2}\right)(z)\right|^{\delta} d z\right)^{\frac{1}{\delta}} \\
& +\left(\frac{1}{|Q|} \int_{Q}\left|\left(b_{1}(z)-\lambda_{1}\right)\left[b_{2}, U^{*}\right]_{2}\left(f_{1}, f_{2}\right)(z)\right|^{\delta} d z\right)^{\frac{1}{\delta}} \\
& +\left(\frac{1}{|Q|} \int_{Q}\left|\left(b_{2}(z)-\lambda_{2}\right)\left[b_{1}, U^{*}\right]_{1}\left(f_{1}, f_{2}\right)(z)\right|^{\delta} d z\right)^{\frac{1}{\delta}} \\
& +\left(\frac{1}{|Q|} \int_{Q} \sup _{\eta>0}\left|U_{\eta}\left(\left(b_{1}-\lambda_{1}\right) f_{1},\left(b_{2}-\lambda_{2}\right) f_{2}\right)(z)-c_{\eta}\right|^{\delta} d z\right)^{\frac{1}{\delta}} \\
\doteq & T_{1}+T_{2}+T_{3}+T_{4} .
\end{aligned}
$$

By Hölder's inequality,

$$
\begin{aligned}
T_{1} & \lesssim \prod_{i=1}^{2}\left\|b_{i}\right\|_{\operatorname{Lip}_{\beta_{i}}}\left(\frac{1}{|Q|^{1-\frac{\delta \beta}{n}}} \int_{Q}\left|U^{*}\left(f_{1}, f_{2}\right)(z)\right|^{\delta} d z\right)^{\frac{1}{\delta}} \\
& \lesssim \prod_{i=1}^{2}\left\|b_{i}\right\|_{\operatorname{Lip}_{\beta_{i}}} M_{\epsilon, \beta}\left(U^{*}\left(f_{1}, f_{2}\right)\right)(x) .
\end{aligned}
$$

In a similar way, we can prove that

$$
T_{2}+T_{3} \lesssim\left\|b_{1}\right\|_{\operatorname{Lip}_{\beta_{1}}} M_{\epsilon, \beta_{1}}\left(\left[b_{2}, U^{*}\right]_{2}\left(f_{1}, f_{2}\right)\right)(x)+\left\|b_{2}\right\|_{\operatorname{Lip}_{\beta_{2}}} M_{\epsilon, \beta_{2}}\left(\left[b_{1}, U^{*}\right]_{1}\left(f_{1}, f_{2}\right)\right)(x)
$$

It remains to estimate the last term $T_{4}$. Take now $c_{\eta}=U_{\eta}\left(\left(b_{1}-\lambda_{1}\right) f_{1}^{\infty},\left(b_{2}-\lambda_{2}\right) f_{2}^{\infty}\right)(x)$.

Then $T_{4} \leq T_{41}+T_{42}+T_{43}+T_{44}$, where

$$
\begin{aligned}
& T_{41}=\left(\frac{1}{|Q|} \int_{Q}\left|U^{*}\left(\left(b_{1}-\lambda_{1}\right) f_{1}^{0},\left(b_{2}-\lambda_{2}\right) f_{2}^{0}\right)(z)\right|^{\delta} d x\right)^{\frac{1}{\delta}} ; \\
& T_{42}=\left(\frac{1}{|Q|} \int_{Q} \sup _{\eta}\left|U_{\eta}\left(\left(b_{1}-\lambda_{1}\right) f_{1}^{0},\left(b_{2}-\lambda_{2}\right) f_{2}^{\infty}\right)(z)\right|^{\delta} d z\right)^{\frac{1}{\delta}} ; \\
& T_{43}=\left(\frac{1}{|Q|} \int_{Q} \sup _{\eta}\left|U_{\eta}\left(\left(b_{1}-\lambda_{1}\right) f_{1}^{\infty},\left(b_{2}-\lambda_{2}\right) f_{2}^{0}\right)(z)\right|^{\delta} d z\right)^{\frac{1}{\delta}} ;
\end{aligned}
$$




$$
\begin{aligned}
T_{44}= & \left(\frac{1}{|Q|} \int_{Q} \sup _{\eta} \mid U_{\eta}\left(\left(b_{1}-\lambda_{1}\right) f_{1}^{\infty},\left(b_{2}-\lambda_{2}\right) f_{2}^{\infty}\right)(z)\right. \\
& \left.-\left.U_{\eta}\left(\left(b_{1}-\lambda_{1}\right) f_{1}^{\infty},\left(b_{2}-\lambda_{2}\right) f_{2}^{\infty}\right)(x)\right|^{\delta} d z\right)^{\frac{1}{\delta}}
\end{aligned}
$$

By the Kolmogorov inequality and by Lemma 3.2,

$$
\begin{aligned}
T_{41} & \lesssim\left\|U^{*}\left(\left(b_{1}-\lambda_{1}\right) f_{1}^{0},\left(b_{2}-\lambda_{2}\right) f_{2}^{0}\right)\right\|_{L^{1 / 2, \infty}\left(Q, \frac{d x}{|Q|}\right)} \\
& \lesssim \frac{1}{|Q|} \int_{Q}\left|\left(b_{1}-\lambda_{1}\right) f_{1}^{0}(z)\right| d z \frac{1}{|Q|} \int_{Q}\left|\left(b_{2}-\lambda_{2}\right) f_{2}^{0}(z)\right| d z \\
& \lesssim \prod_{i=1}^{2}\left\|b_{i}\right\|_{\operatorname{Lip}_{\beta_{i}}} M_{1, \beta_{i}}\left(f_{i}\right)(x) .
\end{aligned}
$$

Next, by Hölder's inequality and by the size condition (1.2),

$$
\begin{aligned}
T_{42} & \leq \frac{1}{|Q|} \int_{Q} \sup _{\eta}\left|U_{\eta}\left(\left(b_{1}-\lambda_{1}\right) f_{1}^{0},\left(b_{2}-\lambda_{2}\right) f_{2}^{\infty}\right)(z)\right| d z \\
& \lesssim \frac{1}{|Q|} \int_{Q} \int_{Q^{*}} \int_{\mathbb{R}^{n} \backslash Q^{*}} \frac{\left|\left(b_{1}\left(y_{1}\right)-\lambda_{1}\right) f_{1}^{0}\left(y_{1}\right)\right|\left|\left(b_{2}\left(y_{2}\right)-\lambda_{2}\right) f_{2}^{\infty}\left(y_{2}\right)\right| d y_{1} d y_{2}}{\left(\left|z-y_{1}\right|+\left|z-y_{2}\right|\right)^{2 n}} d z \\
& \lesssim\left\|b_{1}\right\|_{\operatorname{Lip}_{\beta_{1}}} M_{1, \beta_{1}}\left(f_{1}\right)(x)|Q| \sum_{k=1}^{\infty} \int_{2^{k+3} \sqrt{n} Q \mid 2^{k+2} \sqrt{n} Q} \frac{\left|f_{2}\left(y_{2}\right)\left(b_{2}\left(y_{2}\right)-\lambda_{2}\right)\right| d y_{2}}{\left|y_{2}-x_{Q}\right|^{2 n}} \\
& \lesssim\left\|b_{1}\right\|_{\operatorname{Lip}_{\beta_{1}}} M_{1, \beta_{1}}\left(f_{1}\right)(x)\left\|b_{2}\right\|_{\operatorname{Lip}_{\beta_{2}}} M_{1, \beta_{2}}\left(f_{2}\right)(x) .
\end{aligned}
$$

The operator $T_{43}$ can be estimated in the same way. Finally, we estimate $T_{44}$. By Lemma 3.1 we have

$$
\begin{aligned}
T_{44} \lesssim & \frac{1}{|Q|} \int_{Q} \sup _{\eta} \mid U_{\eta}\left(\left(b_{1}-\lambda_{1}\right) f_{1}^{\infty},\left(b_{2}-\lambda_{2}\right) f_{2}^{\infty}\right)(z) \\
& -U_{\eta}\left(\left(b_{1}-\lambda_{1}\right) f_{1}^{\infty},\left(b_{2}-\lambda_{2}\right) f_{2}^{\infty}\right)(x) \mid d z \\
\lesssim & \frac{1}{|Q|} \int_{Q} \int_{\left(\mathbb{R}^{n} \backslash Q^{*}\right)^{2}} \sup _{\eta}\left|K_{\mu, \eta}(z, \vec{y})-K_{\mu, \eta}\left(x_{Q}, \vec{y}\right)\right| \prod_{i=1}^{2}\left|\left(b_{i}\left(y_{i}\right)-\lambda_{i}\right) f_{i}^{\infty}\left(y_{i}\right)\right| d y_{1} d y_{2} d z \\
\lesssim & \frac{1}{|Q|} \int_{Q} \int_{\left(\mathbb{R}^{n} \backslash Q^{*}\right)^{2}} \frac{1}{\left(\left|x_{Q}-y_{1}\right|+\left|x_{Q}-y_{2}\right|\right)^{2 n}} \omega\left(\frac{\left|z-x_{Q}\right|}{\left|x_{Q}-y_{1}\right|+\left|x_{Q}-y_{2}\right|}\right) \\
& \times \prod_{i=1}^{2}\left|\left(b_{i}\left(y_{i}\right)-\lambda_{i}\right) f_{i}^{\infty}\left(y_{i}\right)\right| d y_{1} d y_{2} d z \\
& +\frac{1}{|Q|} \int_{Q} \int_{\left(\mathbb{R}^{n} \backslash Q^{*}\right)^{2}} \frac{\left|z-x_{Q}\right|}{\left(\left|x_{Q}-y_{1}\right|+\left|x_{Q}-y_{2}\right|\right)^{2 n+1}} \prod_{i=1}^{2}\left|\left(b_{i}\left(y_{i}\right)-\lambda_{i}\right) f_{i}^{\infty}\left(y_{i}\right)\right| d y_{1} d y_{2} d z \\
\lesssim & \frac{1}{|Q|} \int_{Q} \sum_{k=1}^{\infty} \int_{\left(2^{k+3} \sqrt{n} Q 2^{k+2} \sqrt{n} Q\right)^{2}} \frac{1}{\left(\left|2^{k+3} \sqrt{n} Q\right|\right)^{2}} \omega\left(2^{-k}\right) \\
& \times \prod_{i=1}^{2}\left|\left(b_{i}\left(y_{i}\right)-\lambda_{i}\right) f_{i}^{\infty}\left(y_{i}\right)\right| d y_{1} d y_{2} d z
\end{aligned}
$$




$$
\begin{aligned}
& \quad+\frac{1}{|Q|} \int_{Q} \int_{\mathbb{R}^{n} \backslash Q^{*}} \frac{\left|z-x_{Q}\right|^{1 / 2}}{\left|x_{Q}-y_{1}\right|^{n+1 / 2}}\left|\left(b_{1}\left(y_{1}\right)-\lambda_{1}\right) f_{1}^{\infty}\left(y_{1}\right)\right| d y_{1} \\
& \quad \times \int_{\mathbb{R}^{n} \backslash Q^{*}} \frac{\left|z-x_{Q}\right|^{1 / 2}}{\left|x_{Q}-y_{2}\right|^{n+1 / 2}}\left|\left(b_{2}\left(y_{2}\right)-\lambda_{2}\right) f_{2}^{\infty}\left(y_{2}\right)\right| d y_{2} d z \\
& \lesssim \sum_{k=1}^{\infty} \frac{1}{\left(\left|2^{k+3} \sqrt{n} Q\right|\right)^{2}} \int_{\left(2^{k+3} \sqrt{n} Q \backslash 2^{k+2} \sqrt{n} Q\right)^{2}} \omega\left(2^{-k}\right) \prod_{i=1}^{2}\left|\left(b_{i}\left(y_{i}\right)-\lambda_{i}\right) f_{i}^{\infty}\left(y_{i}\right)\right| d \vec{y} \\
& \quad+\sum_{k=1}^{\infty} 2^{-\frac{k}{2}} \frac{1}{\left|2^{k+3} \sqrt{n} Q\right|} \int_{2^{k+3} \sqrt{n} Q \backslash 2^{k+2} \sqrt{n} Q}\left|\left(b_{1}\left(y_{1}\right)-\lambda_{1}\right) f_{1}^{\infty}\left(y_{1}\right)\right| d y_{1} \\
& \quad \times \sum_{k=1}^{\infty} 2^{-\frac{k}{2}} \frac{1}{\left|2^{k+3} \sqrt{n} Q\right|} \int_{2^{k+3} \sqrt{n} Q \backslash 2^{k+2} \sqrt{n} Q}\left|\left(b_{2}\left(y_{2}\right)-\lambda_{2}\right) f_{2}^{\infty}\left(y_{2}\right)\right| d y_{2} \\
& \lesssim\left\|b_{1}\right\|_{\operatorname{Lip}_{\beta_{1}}} M_{1, \beta_{1}}\left(f_{1}\right)(x)\left\|b_{2}\right\|_{\operatorname{Lip}_{\beta_{2}}} M_{1, \beta_{2}}\left(f_{2}\right)(x) .
\end{aligned}
$$

Combining the obtained estimates proves (3.1).

(ii) It is sufficient to prove (3.2) for the operator with only one symbol. Set

$$
\begin{aligned}
U_{\vec{b}}^{* 1}(\vec{f})(x) & =\sup _{\eta>0}\left|b(x) U_{\eta}\left(f_{1}, f_{2}\right)(x)-U_{\eta}\left(b f_{1}, f_{2}\right)(x)\right| \\
& =\sup _{\eta>0}\left|(b(x)-\lambda) U_{\eta}\left(f_{1}, f_{2}\right)(x)-U_{\eta}\left((b-\lambda) f_{1}, f_{2}\right)(x)\right|,
\end{aligned}
$$

where $\lambda=b_{Q^{*}}=\frac{1}{\left|Q^{*}\right|} \int_{Q^{*}} b(y) d y$. Let $c=\sup _{\eta>0}\left|c_{\eta}\right|$. Then

$$
\begin{aligned}
& \left(\left.\frac{1}{|Q|} \int_{Q}|| U_{\vec{b}}^{* 1}\left(f_{1}, f_{2}\right)(z)\right|^{\delta}-|c|^{\delta} \mid d z\right)^{\frac{1}{\delta}} \\
& \quad \lesssim\left(\frac{1}{|Q|} \int_{Q}\left|U_{\vec{b}}^{* 1}\left(f_{1}, f_{2}\right)(z)-\sup _{\eta>0}\right| c_{\eta}||^{\delta} d z\right)^{\frac{1}{\delta}} \\
& \lesssim\left(\frac{1}{|Q|} \int_{Q}\left|(b(z)-\lambda) U^{*}\left(f_{1}, f_{2}\right)(z)\right|^{\delta} d z\right)^{\frac{1}{\delta}} \\
& \quad+\left(\frac{1}{|Q|} \int_{Q} \sup _{\eta>0}\left|U_{\eta}\left((b-\lambda) f_{1}, f_{2}\right)(z)-c_{\eta}\right|^{\delta} d z\right)^{\frac{1}{\delta}} \\
& =: \\
& \left(P_{1}+P_{2}\right) .
\end{aligned}
$$

By Hölder's inequality,

$$
\begin{aligned}
P_{1} & \lesssim\|b\|_{\operatorname{Lip}_{\beta}}\left(\frac{1}{|Q|^{1-\frac{\delta \beta}{n}}} \int_{Q}\left|U^{*}\left(f_{1}, f_{2}\right)(z)\right|^{\delta} d z\right)^{\frac{1}{\delta}} \\
& \lesssim\|b\|_{\operatorname{Lip}_{\beta}} M_{\epsilon, \beta}\left(U^{*}\left(f_{1}, f_{2}\right)\right)(x) .
\end{aligned}
$$

Set $c_{\eta}=U_{\eta}\left((b-\lambda) f_{1}^{\infty}, f_{2}^{\infty}\right)(x)$. Then $P_{2} \leq P_{21}+P_{22}+P_{23}+P_{24}$, where

$$
P_{21}=\left(\frac{1}{|Q|} \int_{Q}\left|U^{*}\left((b-\lambda) f_{1}^{0}, f_{2}^{0}\right)(z)\right|^{\delta} d x\right)^{\frac{1}{\delta}}
$$


Si and Chang Journal of Inequalities and Applications

(2019) 2019:100

Page 12 of 21

$$
\begin{aligned}
& P_{22}=\left(\frac{1}{|Q|} \int_{Q} \sup _{\eta}\left|U_{\eta}\left((b-\lambda) f_{1}^{0}, f_{2}^{\infty}\right)(z)\right|^{\delta} d z\right)^{\frac{1}{\delta}} ; \\
& P_{23}=\left(\frac{1}{|Q|} \int_{Q} \sup _{\eta}\left|U_{\eta}\left((b-\lambda) f_{1}^{\infty}, f_{2}^{0}\right)(z)\right|^{\delta} d z\right)^{\frac{1}{\delta}} ; \\
& P_{24}=\left(\frac{1}{|Q|} \int_{Q} \sup _{\eta}\left|U_{\eta}\left((b-\lambda) f_{1}^{\infty}, f_{2}^{\infty}\right)(z)-U_{\eta}\left((b-\lambda) f_{1}^{\infty}, f_{2}^{\infty}\right)(x)\right|^{\delta} d z\right)^{\frac{1}{\delta}} .
\end{aligned}
$$

By the Kolmogorov inequality and by Lemma 3.2,

$$
\begin{aligned}
P_{21} & \lesssim\left\|U^{*}\left((b-\lambda) f_{1}^{0}, f_{2}^{0}\right)\right\|_{L^{1 / 2, \infty}\left(Q, \frac{d x}{|C|}\right)} \\
& \lesssim \frac{1}{|Q|} \int_{Q}\left|(b-\lambda) f_{1}^{0}(z)\right| d z \frac{1}{|Q|} \int_{Q}\left|f_{2}^{0}(z)\right| d z \\
& \lesssim\|b\|_{\operatorname{Lip}_{\beta}}\left|Q^{*}\right|^{\beta / n} \frac{1}{|Q|} \int_{Q}\left|f_{1}^{0}(z)\right| d z \frac{1}{|Q|} \int_{Q}\left|f_{2}^{0}(z)\right| d z \\
& \lesssim\|b\|_{\operatorname{Lip}_{\beta}} M_{1, \beta}\left(f_{1}\right)(x) M\left(f_{2}\right)(x) .
\end{aligned}
$$

Next, by the size condition (1.2),

$$
\begin{aligned}
P_{22} & \lesssim \frac{1}{|Q|} \int_{Q} \sup _{\eta}\left|U_{\eta}\left((b-\lambda) f_{1}^{0}, f_{2}^{\infty}\right)(z)\right| d z \\
& \lesssim \frac{1}{|Q|} \int_{Q} \int_{Q^{*}} \int_{\left(Q^{*}\right) c} \frac{1}{\left(\left|z-y_{1}\right|+\left|z-y_{2}\right|\right)^{2 n}}\left|\left(b\left(y_{1}\right)-\lambda\right) f_{1}\left(y_{1}\right)\right|\left|f_{2}\left(y_{2}\right)\right| d y_{2} d y_{1} d z \\
& \lesssim \int_{Q^{*}}\left|\left(b\left(y_{1}\right)-\lambda\right) f_{1}\left(y_{1}\right)\right| d y_{1} \int_{\mathbb{R}^{n} \backslash Q^{*}} \frac{\left|f_{2}\left(y_{2}\right)\right| d y_{2}}{\left|x_{Q}-y_{2}\right|^{2 n}} \\
& \lesssim\|b\|_{\operatorname{Lip}_{\beta}}|Q|^{\frac{\beta}{n}} \int_{Q^{*}} f_{1}\left(y_{1}\right) d y_{1} \sum_{k=1}^{\infty} \frac{1}{\left|2^{k+1} Q\right|^{2}} \int_{2^{k+1} Q^{*} \backslash 2^{k} Q^{*}}\left|f_{2}\left(y_{2}\right)\right| d y_{2} \\
& \lesssim\|b\|_{\operatorname{Lip}_{\beta}} M_{1, \beta}\left(f_{1}\right)(x) M\left(f_{2}\right)(x) .
\end{aligned}
$$

Similarly,

$$
P_{23} \lesssim\|b\|_{\operatorname{Lip}_{\beta}} M_{1, \beta}\left(f_{1}\right)(x) M\left(f_{2}\right)(x) .
$$

By Lemma 3.1 we obtain

$$
\begin{aligned}
P_{24} & \lesssim \frac{1}{|Q|} \int_{Q} \sup _{\eta}\left|U_{\eta}\left((b-\lambda) f_{1}^{\infty}, f_{2}^{\infty}\right)(z)-U_{\eta}\left((b-\lambda) f_{1}^{\infty}, f_{2}^{\infty}\right)(x)\right| d z \\
& \lesssim \frac{1}{|Q|} \int_{Q} \int_{\left(\mathbb{R}^{n} \backslash Q^{*}\right)^{2}} \sup _{\eta}\left|K_{\mu, \eta}(z, \vec{y})-K_{\mu, \eta}\left(x_{Q}, \vec{y}\right)\right|\left|\left(b\left(y_{1}\right)-\lambda\right)\right| \prod_{i=1}^{2}\left|f_{i}^{\infty}\left(y_{i}\right)\right| d y_{1} d y_{2} d z \\
& \lesssim \frac{1}{|Q|} \int_{Q} \int_{\left(\mathbb{R}^{n} \backslash Q^{*}\right)^{2}} \frac{\omega\left(\frac{\left|z-x_{Q}\right|}{\left|z-y_{1}\right|+\left|z-y_{2}\right|}\right)}{\left(\left|z-y_{1}\right|+\left|z-y_{2}\right|\right)^{2 n}}\left|\left(b\left(y_{1}\right)-\lambda\right)\right| \prod_{i=1}^{2}\left|f_{i}^{\infty}\left(y_{i}\right)\right| d y_{1} d y_{2} d z \\
& +\frac{1}{|Q|} \int_{Q} \int_{\left(\mathbb{R}^{n} \backslash Q^{*}\right)^{2}} \frac{\left|z-x_{Q}\right|}{\left(\left|z-y_{1}\right|+\left|z-y_{2}\right|\right)^{2 n+1}}\left|\left(b\left(y_{1}\right)-\lambda\right)\right| \prod_{i=1}^{2}\left|f_{i}^{\infty}\left(y_{i}\right)\right| d y_{1} d y_{2} d z
\end{aligned}
$$




$$
\begin{aligned}
& \lesssim \frac{1}{|Q|} \int_{Q} \sum_{k=1}^{\infty} \int_{\left(2^{k+3} \sqrt{n} Q \mid 2^{k+2} \sqrt{n} Q\right)^{2}} \frac{1}{\left(\left|2^{k+3} \sqrt{n} Q\right|\right)^{2}} \omega\left(2^{-k}\right)\left|\left(b\left(y_{1}\right)-\lambda\right)\right| \\
& \quad \times \prod_{i=1}^{2}\left|f_{i}^{\infty}\left(y_{i}\right)\right| d y_{1} d y_{2} d z+\frac{1}{|Q|} \int_{Q} \int_{\mathbb{R}^{n} \backslash Q^{*}} \frac{\left|z-x_{Q}\right|^{1 / 2}}{\left|x_{Q}-y_{1}\right|^{n+1 / 2}}\left|\left(b\left(y_{1}\right)-\lambda\right) f_{1}^{\infty}\left(y_{1}\right)\right| d y_{1} \\
& \quad \times \int_{\mathbb{R}^{n} \backslash Q^{*}} \frac{\left|z-x_{Q}\right|^{1 / 2}}{\left|x_{Q}-y_{2}\right|^{n+1 / 2}}\left|f_{2}^{\infty}\left(y_{2}\right)\right| d y_{2} d z \\
& \lesssim\|b\|_{\operatorname{Lip}_{\beta}} \sum_{k=1}^{\infty} \frac{\omega\left(2^{-k}\right)}{\left(\left|2^{k+3} \sqrt{n} Q\right|\right)^{1-\beta / n}} \int_{2^{k+3} \sqrt{n} Q}\left|f_{1}^{\infty}\left(y_{1}\right)\right| d y_{1} \frac{1}{\left|2^{k} Q^{*}\right|} \int_{2^{k+3} \sqrt{n} Q}\left|f_{2}^{\infty}\left(y_{2}\right)\right| d y_{2} \\
& \quad+\sum_{k=1}^{\infty} 2^{-\frac{k}{2}} \frac{1}{\left|2^{k+3} \sqrt{n} Q\right|^{1-\beta / n}} \int_{2^{k+3} \sqrt{n} Q \backslash 2^{k+2} \sqrt{n} Q}\left|f_{1}^{\infty}\left(y_{1}\right)\right| d y_{1} \\
& \quad \times \sum_{k=1}^{\infty} 2^{-\frac{k}{2}} \frac{1}{\left|2^{k+3} \sqrt{n} Q\right|} \int_{2^{k+3} \sqrt{n} Q \backslash 2^{k+2} \sqrt{n} Q}\left|f_{2}^{\infty}\left(y_{2}\right)\right| d y_{2} \\
& \lesssim\|b\|_{\operatorname{Lip}_{\beta} M_{1, \beta}\left(f_{1}\right)(x) M\left(f_{2}\right)(x) .}
\end{aligned}
$$

Thus we finish the proof of (3.2). Then Lemma 3.3 is proved.

\section{Proofs of Theorems 1.2 and 1.3}

The main ideas in this section are from [17] and [19]. We should also mention that the proof of this part is similar to that of Theorem 1.2 and Theorem 1.3 in [22]; we just give the different part of the proof.

We begin with the proof of Theorem 1.2.

Proof For any cube $Q$ centered at $x_{Q}$, the theorem will be proved if we can show that

$$
\sup _{Q} \frac{1}{|Q|^{1+\beta / n-1 / p}} \int_{Q}\left|U_{\Pi \vec{b}}^{*}(\vec{f})(z)-\left(U_{\Pi \vec{b}}^{*}(\vec{f})\right)_{Q}\right| d z \lesssim\left\|b_{1}\right\|_{{\dot{\beta_{1}}}_{1}}\left\|b_{2}\right\|_{{\dot{\beta_{2}}}_{2}}\left\|f_{1}\right\|_{L^{p_{1}}}\left\|f_{2}\right\|_{L^{p_{2}}}
$$

Set $c=c_{1}+c_{2}+c_{3}$, which will be determined later. We estimate

$$
\begin{aligned}
\frac{1}{|Q|^{1+\beta / n-1 / p}} \int_{Q}\left|U_{\Pi \vec{b}}^{*}(\vec{f})(z)-\left(U_{\Pi \vec{b}}^{*}(\vec{f})\right)_{Q}\right| d z \\
\lesssim \frac{1}{|Q|^{1+\beta / n-1 / p}} \int_{Q}\left|U_{\Pi \vec{b}}^{*}\left(f_{1}, f_{2}\right)(z)-c\right| d z \\
\lesssim \frac{1}{|Q|^{1+\beta / n-1 / p}} \int_{Q}\left|U_{\Pi \vec{b}}^{*}\left(f_{1}^{0}, f_{2}^{0}\right)(z)\right| d z \\
\quad+\frac{1}{|Q|^{1+\beta / n-1 / p}} \int_{Q}\left|U_{\Pi \vec{b}}^{*}\left(f_{1}^{0}, f_{2}^{\infty}\right)(z)-c_{1}\right| d z \\
\quad+\frac{1}{|Q|^{1+\beta / n-1 / p}} \int_{Q}\left|U_{\Pi \vec{b}}^{*}\left(f_{1}^{\infty}, f_{2}^{0}\right)(z)-c_{2}\right| d z \\
\quad+\frac{1}{|Q|^{1+\beta / n-1 / p}} \int_{Q}\left|U_{\Pi \vec{b}}^{*}\left(f_{1}^{\infty}, f_{2}^{\infty}\right)(z)-c_{3}\right| d z \\
\doteq M_{1}+M_{2}+M_{3}+M_{4} .
\end{aligned}
$$


We estimate these terms separately. For the first term, we can choose $1<q, q_{j}<\infty$, $q_{j}<n / \beta_{j}<p_{j}, j=1,2$, with $1 / q=1 / q_{1}+1 / q_{2}-\left(\beta_{1}+\beta_{2}\right) / n$. By Hölder's inequality and by Theorem 1.1 we have

$$
\begin{aligned}
M_{1} & \lesssim \frac{1}{|Q|^{1+\beta / n-1 / p}}\left(\int_{Q}\left|U_{\Pi \vec{b}}^{*}\left(f_{1}^{0}, f_{2}^{0}\right)(z)\right|^{q} d z\right)^{1 / q}|Q|^{1-1 / q} \\
& \lesssim \frac{1}{|Q|^{1+\beta / n-1 / p}}|Q|^{1-1 / q}\left\|f_{1}^{0}\right\|_{L^{q_{1}}}\left\|f_{2}^{0}\right\|_{L^{q_{2}}} \\
& \lesssim\left\|f_{1}\right\|_{L^{p_{1}}\left\|f_{2}\right\|_{L^{p_{2}}} .}
\end{aligned}
$$

To get $M_{2}$, we take $c_{1}=T\left(\left(b_{1}-\lambda_{1}\right) f_{1}^{0}, f_{2}^{\infty}\right)\left(x_{Q}\right)$. Then

$$
\begin{aligned}
M_{2} \lesssim & \frac{1}{|Q|^{1+\beta / n-1 / p}} \int_{Q} \sup _{\eta} \mid \int_{Q^{*}} \int_{\mathbb{R}^{n} \backslash Q^{*}}\left(b_{1}(z)-\lambda_{1}\right)\left(b_{2}(z)-\lambda_{2}\right) \\
& \times K_{\mu, \eta}\left(z, y_{1}, y_{2}\right) f_{1}\left(y_{1}\right) f_{2}\left(y_{2}\right) d y_{1} d y_{2} \mid d z \\
& +\frac{1}{|Q|^{1+\beta / n-1 / p}} \int_{Q} \sup \mid \int_{Q^{*}} \int_{\mathbb{R}^{n} \backslash Q^{*}}\left(b_{1}(z)-\lambda_{1}\right)\left(b_{2}\left(y_{2}\right)-\lambda_{2}\right) \\
& \times K_{\mu, \eta}\left(z, y_{1}, y_{2}\right) f_{1}\left(y_{1}\right) f_{2}\left(y_{2}\right) d y_{1} d y_{2} \mid d z \\
& +\frac{1}{|Q|^{1+\beta / n-1 / p}} \int_{Q} \sup \mid \int_{Q^{*}} \int_{\mathbb{R}^{n} \backslash Q^{*}}\left(b_{1}\left(y_{1}\right)-\lambda_{1}\right)\left(b_{2}(z)-\lambda_{2}\right) \\
& \times K_{\mu, \eta}\left(z, y_{1}, y_{2}\right) f_{1}\left(y_{1}\right) f_{2}\left(y_{2}\right) d y_{1} d y_{2} \mid d z \\
& +\frac{1}{|Q|^{1+\beta / n-1 / p}} \int_{Q} \sup _{\eta} \mid \int_{Q^{*}} \int_{\mathbb{R}^{n} \backslash Q^{*}}\left(b_{1}\left(y_{1}\right)-\lambda_{1}\right)\left(b_{2}\left(y_{2}\right)-\lambda_{2}\right) \\
& \times\left[K_{\mu, \eta}\left(z, y_{1}, y_{2}\right)-K_{\mu, \eta}\left(x_{Q}, y_{1}, y_{2}\right)\right] f_{1}\left(y_{1}\right) f_{2}\left(y_{2}\right) d y_{1} d y_{2} \mid d z \\
\doteq & M_{21}+M_{22}+M_{23}+M_{24} .
\end{aligned}
$$

Using the size condition (1.2) and the estimate in [22, p. 5013], we have

$$
\begin{aligned}
M_{21} \lesssim & \frac{1}{|Q|^{1+\beta / n-1 / p}} \int_{Q} \int_{Q^{*}} \int_{\mathbb{R}^{n} \backslash Q^{*}}\left|\left(b_{1}(z)-\lambda_{1}\right)\left(b_{2}(z)-\lambda_{2}\right)\right| \\
& \times \frac{1}{\left(\left|z-y_{1}\right|+\left|z-y_{2}\right|\right)^{2 n}}\left|f_{1}\left(y_{1}\right) f_{2}\left(y_{2}\right)\right| d y_{1} d y_{2} d z \\
\lesssim & \left\|b_{1}\right\|{\dot{\lambda_{\beta}}}_{\beta_{1}}\left\|b_{2}\right\|_{\dot{\lambda}_{\beta_{2}}}\left\|f_{1}\right\|_{L^{p_{1}}}\left\|f_{2}\right\|_{L^{p_{2}}} .
\end{aligned}
$$

In a similar way, we get $M_{23}+M_{22} \lesssim\left\|b_{1}\right\|_{{\dot{\beta_{1}}}_{1}}\left\|b_{2}\right\|_{{\dot{\beta_{2}}}_{2}}\left\|f_{1}\right\|_{L^{p_{1}}}\left\|f_{2}\right\|_{L^{p_{2}}}$.

By Minkowski's inequality and by Lemma 3.1,

$$
\begin{aligned}
M_{24} \lesssim & \frac{1}{|Q|^{1+\beta / n-1 / p}} \int_{Q} \sup _{\eta} \int_{Q^{*}} \int_{\mathbb{R}^{n} \backslash Q^{*}}\left|\left(b_{1}\left(y_{1}\right)-\lambda_{1}\right)\left(b_{2}\left(y_{2}\right)-\lambda_{2}\right)\right| \\
& \times\left|K_{\mu, \eta}\left(z, y_{1}, y_{2}\right)-K_{\mu, \eta}\left(x_{Q}, y_{1}, y_{2}\right)\right|\left|f_{1}\left(y_{1}\right) f_{2}\left(y_{2}\right)\right| d y_{1} d y_{2} d z
\end{aligned}
$$




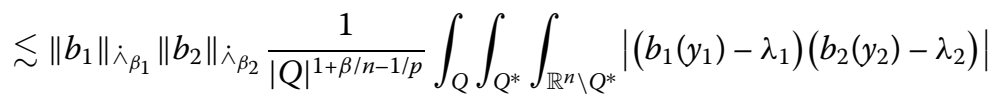

$$
\begin{aligned}
& \times\left(\frac{\omega\left(\frac{\left|z-x_{Q}\right|}{\left|z-y_{1}\right|+\left|z-y_{2}\right|}\right)}{\left(\left|z-y_{1}\right|+\left|z-y_{2}\right|\right)^{2 n}}+\frac{\left|z-x_{Q}\right|}{\left(\left|x-y_{1}\right|+\left|x-y_{2}\right|\right)^{2 n+1}}\right)\left|f_{1}\left(y_{1}\right) f_{2}\left(y_{2}\right)\right| d y_{1} d y_{2} d z \\
& \lesssim\left\|b_{1}\right\|_{{\dot{\beta_{1}}}_{1}}\left\|b_{2}\right\|_{\dot{\lambda}_{\beta_{2}}} \frac{1}{|Q|^{1+\beta_{2} / n-1 / p}} \\
& \times \int_{Q} \int_{Q^{*}} \int_{\mathbb{R}^{n} \backslash Q^{*}}\left(\frac{\omega\left(\frac{\left|z-x_{Q}\right|}{\left|x_{Q}-y_{2}\right|}\right)}{\left(\left|z-y_{1}\right|+\left|z-y_{2}\right|\right)^{2 n-\beta_{2}}}+\frac{2^{-k}}{\left(\left|z-y_{1}\right|+\left|z-y_{2}\right|\right)^{2 n-\beta_{2}}}\right) \\
& \times\left|f_{1}\left(y_{1}\right) f_{2}\left(y_{2}\right)\right| d y_{1} d y_{2} d z \\
& \lesssim\left\|b_{1}\right\|_{{\dot{\beta_{1}}}_{1}}\left\|b_{2}\right\|_{{\dot{\lambda}}_{\beta_{2}}} \frac{1}{|Q|^{1+\beta_{2} / n-1 / p}} \\
& \times \int_{Q} \int_{Q^{*}} \int_{\mathbb{R}^{n} \backslash Q^{*}} \frac{\omega\left(2^{-k}\right)+2^{-k}}{\left(\left|z-y_{1}\right|+\left|z-y_{2}\right|\right)^{2 n-\beta_{2}}}\left|f_{1}\left(y_{1}\right) f_{2}\left(y_{2}\right)\right| d y_{1} d y_{2} d z \\
& \lesssim \frac{\left\|b_{1}\right\|_{\dot{\lambda}_{\beta_{1}}}\left\|b_{2}\right\|_{{\dot{\beta_{2}}}_{2}}}{|Q|^{\beta_{2} / n-1 / p}} \int_{Q^{*}}\left|f_{1}\left(y_{1}\right)\right| d y_{1} \sum_{k=1}^{\infty} \frac{\omega\left(2^{-k}\right)+2^{-k}}{\left|2^{k+3} \sqrt{n} Q\right|^{2-\beta_{2} / n}} \\
& \times \int_{2^{k+3} \sqrt{n} Q \backslash 2^{k+2} \sqrt{n} Q}\left|f_{2}\left(y_{2}\right)\right| d y_{2} \\
& \lesssim\left\|b_{1}\right\|{\dot{\lambda}_{\beta_{1}}}_{\| b_{2}}\left\|_{{\dot{\beta_{2}}}_{2}}\right\| f_{1}\left\|_{L^{p_{1}}}\right\| f_{2} \|_{L^{p_{2}}} \sum_{k=1}^{\infty}\left(\omega\left(2^{-k}\right)+2^{-k}\right) 2^{-k n\left(1-\beta_{2} / n+1 / p_{2}\right)} \\
& \lesssim\left\|b_{1}\right\|_{{\dot{\lambda_{1}}}_{1}}\left\|b_{2}\right\|_{{\dot{\beta_{2}}}_{2}}\left\|f_{1}\right\|_{L^{p_{1}}}\left\|f_{2}\right\|_{L^{p_{2}}},
\end{aligned}
$$

where we have used assumption (1.5) and the inequality $1-\beta_{2} / n+1 / p_{2}>0$.

Thus $M_{2} \lesssim\left\|b_{1}\right\|_{{\dot{\beta_{1}}}_{1}}\left\|b_{2}\right\|_{{\dot{\beta_{2}}}_{2}}\left\|f_{1}\right\|_{L^{p_{1}}}\left\|f_{2}\right\|_{L^{p_{2}}}$. Similarly, $M_{3} \lesssim\left\|b_{1}\right\|_{{\dot{\beta}}_{1}}\left\|b_{2}\right\|{{\dot{\lambda}}_{\beta_{2}}}_{\| f_{1}}\left\|_{L^{p_{1}}}\right\| f_{2} \|_{L^{p_{2}}}$.

We deal with $M_{4}$ as follows:

$$
\begin{aligned}
M_{4} \lesssim & \frac{1}{|Q|^{1+\beta / n-1 / p}} \int_{Q} \sup _{\eta} \mid \int_{\left(\mathbb{R}^{n} \backslash Q^{*}\right)^{2}}\left(b_{1}(z)-\lambda_{1}\right)\left(b_{2}(z)-\lambda_{2}\right) \\
& \times K_{\mu, \eta}\left(z, y_{1}, y_{2}\right) f_{1}\left(y_{1}\right) f_{2}\left(y_{2}\right) d y_{1} d y_{2} \mid d z \\
& +\frac{1}{|Q|^{1+\beta / n-1 / p}} \int_{Q} \sup _{\eta} \mid \int_{\left(\mathbb{R}^{n} \backslash Q^{*}\right)^{2}}\left(b_{1}(z)-\lambda_{1}\right)\left(b_{2}\left(y_{2}\right)-\lambda_{2}\right) \\
& \times\left[K_{\mu, \eta}\left(z, y_{1}, y_{2}\right)-K_{\mu, \eta}\left(x_{Q}, y_{1}, y_{2}\right)\right] f_{1}\left(y_{1}\right) f_{2}\left(y_{2}\right) d y_{1} d y_{2} \mid d z \\
& +\frac{1}{|Q|^{1+\beta / n-1 / p}} \int_{Q} \sup _{\eta} \mid \int_{\left(\mathbb{R}^{n} \backslash Q^{*}\right)^{2}}\left(b_{1}\left(y_{1}\right)-\lambda_{1}\right)\left(b_{2}(z)-\lambda_{2}\right) \\
& \times\left[K_{\mu, \eta}\left(z, y_{1}, y_{2}\right)-K_{\mu, \eta}\left(x_{Q}, y_{1}, y_{2}\right)\right] f_{1}\left(y_{1}\right) f_{2}\left(y_{2}\right) d y_{1} d y_{2} \mid d z \\
& +\frac{1}{|Q|^{1+\beta / n-1 / p}} \int_{Q} \sup _{\eta} \mid \int_{\left(\mathbb{R}^{n} \backslash Q^{*}\right)^{2}}\left(b_{1}\left(y_{1}\right)-\lambda_{1}\right)\left(b_{2}\left(y_{2}\right)-\lambda_{2}\right) \\
& \times\left[K_{\mu, \eta}\left(z, y_{1}, y_{2}\right)-K_{\mu, \eta}\left(x_{Q}, y_{1}, y_{2}\right)\right] f_{1}\left(y_{1}\right) f_{2}\left(y_{2}\right) d y_{1} d y_{2} \mid d z \\
\doteq & M_{41}+M_{42}+M_{43}+M_{44} .
\end{aligned}
$$


By Minkowski's inequality and by the size condition (1.2),

$$
\begin{aligned}
M_{41} \lesssim & \frac{1}{|Q|^{1+\beta / n-1 / p}} \int_{Q} \sup _{\eta} \int_{\left(\mathbb{R}^{n} \backslash Q^{*}\right)^{2}}\left|\left(b_{1}(z)-\lambda_{1}\right)\left(b_{2}(z)-\lambda_{2}\right)\right| \\
& \times\left|K_{\mu, \eta}\left(z, y_{1}, y_{2}\right)\right|\left|f_{1}\left(y_{1}\right) f_{2}\left(y_{2}\right)\right| d y_{1} d y_{2} d z \\
\lesssim & \frac{1}{|Q|^{1+\beta / n-1 / p}} \int_{Q} \int_{\left(\mathbb{R}^{n} \backslash Q^{*}\right)^{2}}\left|\left(b_{1}(z)-\lambda_{1}\right)\left(b_{2}(z)-\lambda_{2}\right)\right| \\
& \times \frac{1}{\left(\left|z-y_{1}\right|+\left|z-y_{2}\right|\right)^{2 n}}\left|f_{1}\left(y_{1}\right) f_{2}\left(y_{2}\right)\right| d y_{1} d y_{2} d z \\
\lesssim & \left\|b_{1}\right\|_{\lambda_{\beta_{1}}}\left\|b_{2}\right\|_{\lambda_{\beta_{2}}} \mid f_{1}\left\|_{L^{p_{1}}}\right\| f_{2} \|_{L^{p_{2}}} .
\end{aligned}
$$

Using Minkowski's inequality along with Lemma 3.1, we obtain

$$
\begin{aligned}
M_{42} \lesssim & \frac{1}{|Q|^{1+\beta / n-1 / p}} \int_{Q} \sup _{\eta} \int_{\left(\mathbb{R}^{n} \backslash Q^{*}\right)^{2}}\left|\left(b_{1}(z)-\lambda_{1}\right)\left(b_{2}\left(y_{2}\right)-\lambda_{2}\right)\right| \\
& \times\left|K_{\mu, \eta}\left(z, y_{1}, y_{2}\right)-K_{\mu, \eta}\left(x_{Q}, y_{1}, y_{2}\right)\right|\left|f_{1}\left(y_{1}\right) f_{2}\left(y_{2}\right)\right| d y_{1} d y_{2} d z \\
\lesssim & \frac{\left\|b_{1}\right\|_{\dot{\lambda}_{\beta_{1}}}\left\|b_{2}\right\|_{\dot{\lambda}_{\beta_{2}}}}{|Q|^{\beta_{2} / n-1 / p}} \sum_{k=1}^{\infty} \int_{2^{k+3} \sqrt{n} Q 2^{k+2} \sqrt{n} Q} \frac{\left|f_{1}\left(y_{1}\right)\right|}{\left|y_{1}-x_{Q}\right|^{n}} d y_{1} \\
& \times \sum_{k=1}^{\infty} \int_{2^{k+3} \sqrt{n} Q \backslash 2^{k+2} \sqrt{n} Q} \frac{\left|f_{2}\left(y_{2}\right)\right|\left(\omega\left(2^{-k}\right)+\frac{\left|z-x_{Q}\right|}{\left|x_{Q}-y_{1}\right|+\left|x_{Q}-y_{2}\right|}\right)}{\left|y_{2}-x_{Q}\right|^{n-\beta_{2}}} d y_{2} \\
\lesssim & \frac{\left\|b_{1}\right\|_{\dot{\lambda}_{\beta_{1}}}\left\|b_{2}\right\|_{\dot{\beta}_{\beta_{2}}}}{|Q|^{\beta_{2} / n-1 / p}} \sum_{k=1}^{\infty} \frac{1}{\left|2^{k+3} \sqrt{n} Q\right|} \int_{2^{k+3} \sqrt{n} Q} f_{1}\left(y_{1}\right) d y_{1} \\
& \times \sum_{k=1}^{\infty}\left(\omega\left(2^{-k}\right)+2^{-k}\right) \frac{1}{\left|2^{k+3} \sqrt{n} Q\right|^{1-\beta_{2} / n}} \int_{2^{k+3} \sqrt{n} Q} f_{2}\left(y_{2}\right) d y_{2} \\
\lesssim & \left\|b_{1}\right\|_{\dot{\beta}_{\beta_{1}}}\left\|b_{2}\right\|_{\dot{\lambda}_{\beta_{2}}}\left\|f_{1}\right\|_{L^{p_{1}}}\left\|f_{2}\right\|_{L^{p_{2}}} \sum_{k=1}^{\infty}\left(\omega\left(2^{-k}\right)+2^{-k}\right) 2^{k n\left(\beta_{2} / n-1 / p\right)} \\
\lesssim & \left\|b_{1}\right\|_{\dot{\lambda}_{\beta_{1}}}\left\|b_{2}\right\|_{\dot{\lambda}_{\beta_{2}}}\left\|f_{1}\right\|_{L^{p_{1}}}\left\|f_{2}\right\|_{L^{p_{2}}},
\end{aligned}
$$

where we have used assumption (1.5) and the inequality $0<\beta-n / p<1$.

Similarly, $M_{43} \lesssim\left\|b_{1}\right\|_{{\dot{\beta_{1}}}_{1}}\left\|b_{2}\right\|_{{\dot{\beta_{2}}}_{\beta_{2}}}\left\|f_{1}\right\|_{L^{p_{1}}}\left\|f_{2}\right\|_{L^{p_{2}}}$.

Now we estimate $M_{44}$ :

$$
\begin{aligned}
M_{44} \lesssim & \frac{1}{|Q|^{1+\beta / n-1 / p}} \int_{Q} \sup _{\eta} \int_{\left(\mathbb{R}^{n} \backslash Q^{*}\right)^{2}}\left|\left(b_{1}\left(y_{1}\right)-\lambda_{1}\right)\left(b_{2}\left(y_{2}\right)-\lambda_{2}\right)\right| \\
& \times\left|K_{\mu, \eta}\left(z, y_{1}, y_{2}\right)-K_{\mu, \eta}\left(x_{Q}, y_{1}, y_{2}\right)\right|\left|f_{1}\left(y_{1}\right) f_{2}\left(y_{2}\right)\right| d y_{1} d y_{2} d z \\
\lesssim & \frac{\left\|b_{1}\right\|{\dot{\lambda_{1}}}_{\beta_{1}}|| b_{2} \|{\dot{\lambda_{\beta}}}_{\beta_{2}}}{|Q|^{1+\beta / n-1 / p}} \int_{Q} \sum_{k=1}^{\infty} \int_{\left(2^{k+3} \sqrt{n} Q\right)^{2} \backslash\left(2^{k+2} \sqrt{n} Q\right)^{2}} \frac{\left|f_{1}\left(y_{1}\right)\right|}{\left|y_{2}-x_{Q}\right|^{2 n-\beta_{1}-\beta_{2}}} \\
& \times\left(\omega\left(\frac{\left|z-x_{Q}\right|}{\left|y_{2}-x_{Q}\right|}\right)+\frac{\left|z-x_{Q}\right|}{\left|x_{Q}-y_{1}\right|+\left|x_{Q}-y_{2}\right|}\right) d y_{1} d y_{2} d z
\end{aligned}
$$




$$
\begin{aligned}
& \lesssim\left\|b_{1}\right\|_{{\dot{\beta_{1}}}_{1}}\left\|b_{2}\right\|_{{\dot{\beta_{2}}}_{2}}\left\|f_{1}\right\|_{L^{p_{1}}}\left\|f_{2}\right\|_{L^{p_{2}}} \sum_{k=1}^{\infty}\left(\omega\left(2^{-k}\right)+2^{-k}\right) 2^{k n(\beta / n-1 / p)} \\
& \lesssim\left\|b_{1}\right\|{\dot{\dot{\beta}_{1}}}_{\|}\left\|b_{2}\right\|{\dot{\lambda_{\beta_{2}}}}_{\|}\left\|f_{1}\right\|_{L^{p_{1}}}\left\|f_{2}\right\|_{L^{p_{2}}} .
\end{aligned}
$$

Putting the estimates for $M_{1}, M_{2}, M_{3}, M_{4}$ together, we get (4.1). Thus the proof of Theorem 1.2 is completed.

\section{Proof of Theorem 1.3.}

Proof We use the same notations as in previous sections. Then we have

$$
\begin{aligned}
& \frac{1}{|Q|^{1+\beta / n}} \int_{Q}\left|U_{\Pi \vec{b}}^{*}(\vec{f})(z)-\left(U_{\Pi \vec{b}}^{*}(\vec{f})\right)_{Q}\right| d z \\
& \lesssim \frac{1}{|Q|^{1+\beta / n}} \int_{Q}\left|\left(b_{1}(z)-\lambda_{1}\right)\left(b_{2}(z)-\lambda_{2}\right) U^{*}\left(f_{1}, f_{2}\right)(z)\right| d z \\
& \quad+\frac{1}{|Q|^{1+\beta / n}} \int_{Q}\left|\left(b_{2}(z)-\lambda_{2}\right) U_{\vec{b}}^{*, 1}\left(f_{1}, f_{2}\right)(z)-c_{1}\right| d z \\
& \quad+\frac{1}{|Q|^{1+\beta / n}} \int_{Q}\left|\left(b_{1}(z)-\lambda_{1}\right) U_{\vec{b}}^{*, 2}\left(f_{1}, f_{2}\right)(z)-c_{2}\right| d z \\
& \quad+\frac{1}{|Q|^{1+\beta / n}} \int_{Q}\left|U^{*}\left(\left(b_{1}-\lambda_{1}\right) f_{1},\left(b_{2}-\lambda_{2}\right) f_{2}\right)(z)-c_{3}\right| d z \\
& \doteq N_{1}+N_{2}+N_{3}+N_{4} .
\end{aligned}
$$

For $1<r<p$, by the Hölder inequality, we have

$$
N_{1} \lesssim\left\|b_{1}\right\|_{\dot{\lambda}_{\beta_{1}}}\left\|b_{2}\right\|_{\dot{\lambda}_{\beta_{2}}} M_{r}\left(U^{*}\left(f_{1}, f_{2}\right)\right)(x)
$$

In what follows, we just give an estimate for $N_{2}$, since $N_{3}$ and $N_{4}$ can be estimated in a similar way. Let

$$
\begin{aligned}
& c_{1}^{\prime}=\left\|b_{2}\right\|{\dot{\lambda_{\beta_{2}}}}_{\mid}|Q|^{\beta_{2} / n} \sup _{\eta}\left|\int_{\left(\mathbb{R}^{n}\right)^{2}}\left(b_{1}\left(y_{1}\right)-\lambda_{1}\right) K_{\mu, \eta}\left(x_{Q}, y_{1}, y_{2}\right) f_{1}^{\infty}\left(y_{1}\right) f_{2}^{0}\left(y_{2}\right) d y_{1} d y_{2}\right|
\end{aligned}
$$

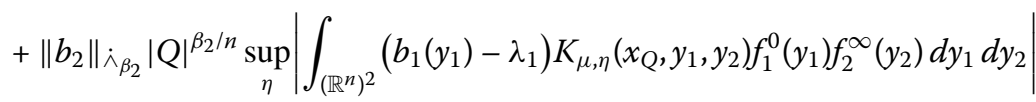

$$
\begin{aligned}
& +\left\|b_{2}\right\|{\dot{\lambda_{\beta}}}_{\beta_{2}}|Q|^{\beta_{2} / n} \sup _{\eta}\left|\int_{\left(\mathbb{R}^{n}\right)^{2}}\left(b_{1}\left(y_{1}\right)-\lambda_{1}\right) K_{\mu, \eta}\left(x_{Q}, y_{1}, y_{2}\right) f_{1}^{\infty}\left(y_{1}\right) f_{2}^{\infty}\left(y_{2}\right) d y_{1} d y_{2}\right| \text {. }
\end{aligned}
$$

Observe that

$$
\begin{aligned}
U_{\vec{b}}^{*, 1}\left(f_{1}, f_{2}\right)(z)< & \left|\left(b_{1}(z)-\lambda_{1}\right)\right| U^{*}\left(f_{1}, f_{2}\right)(z)+U^{*}\left(\left(b_{1}-\lambda_{1}\right) f_{1}^{0}, f_{2}^{0}\right)(z) \\
& +\sup _{\eta}\left|\int_{\left(\mathbb{R}^{n}\right)^{2}}\left(b_{1}\left(y_{1}\right)-\lambda_{1}\right) K_{\mu, \eta}\left(x, y_{1}, y_{2}\right) f_{1}^{\infty}\left(y_{1}\right) f_{2}^{0}\left(y_{2}\right) d y_{1} d y_{2}\right| \\
& +\sup _{\eta}\left|\int_{\left(\mathbb{R}^{n}\right)^{2}}\left(b_{1}\left(y_{1}\right)-\lambda_{1}\right) K_{\mu, \eta}\left(x, y_{1}, y_{2}\right) f_{1}^{0}\left(y_{1}\right) f_{2}^{\infty}\left(y_{2}\right) d y_{1} d y_{2}\right| \\
& +\sup _{\eta}\left|\int_{\left(\mathbb{R}^{n}\right)^{2}}\left(b_{1}\left(y_{1}\right)-\lambda_{1}\right) K_{\mu, \eta}\left(x, y_{1}, y_{2}\right) f_{1}^{\infty}\left(y_{1}\right) f_{2}^{\infty}\left(y_{2}\right) d y_{1} d y_{2}\right| .
\end{aligned}
$$


From this we have

$$
\begin{aligned}
& \left.N_{2} \lesssim \frac{1}{|Q|^{1+\beta / n}} \int_{Q}\left|\left\|b_{2}\right\|_{\lambda_{\beta_{2}}}\right| Q\right|^{\beta_{2} / n} U_{\vec{b}}^{*, 1}\left(f_{1}, f_{2}\right)(z)-c_{1}^{\prime} \mid d z \\
& \lesssim \frac{\left\|b_{2}\right\|_{{\dot{\beta_{2}}}_{2}}}{|Q|^{1+\beta_{1} / n}} \int_{Q}\left|\left(b_{1}(z)-\lambda_{1}\right)\right| U^{*}\left(f_{1}, f_{2}\right)(z) d z+\frac{\left\|b_{2}\right\|_{\dot{\lambda}_{\beta_{2}}}}{|Q|^{1+\beta_{1} / n}} \int_{Q} U^{*}\left(\left(b_{1}-\lambda_{1}\right) f_{1}^{0}, f_{2}^{0}\right)(z) d z \\
& +\frac{\left\|b_{2}\right\|{\dot{\lambda_{\beta_{2}}}}_{\mathrm{C}^{1}}}{|Q|^{1+\beta_{1} / n}} \int_{Q} \sup \mid \int_{\left.\mathbb{R}^{n}\right)^{2}}\left(b_{1}\left(y_{1}\right)-\lambda_{1}\right) \\
& \times\left[K_{\mu, \eta}\left(z, y_{1}, y_{2}\right)-K_{\mu, \eta}\left(x_{Q}, y_{1}, y_{2}\right)\right] f_{1}^{0}\left(y_{1}\right) f_{2}^{\infty}\left(y_{2}\right) d y_{1} d y_{2} \mid d z
\end{aligned}
$$

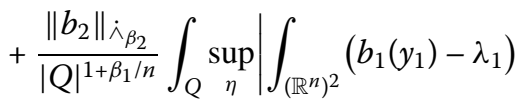

$$
\begin{aligned}
& \times\left[K_{\mu, \eta}\left(z, y_{1}, y_{2}\right)-K_{\mu, \eta}\left(x_{Q}, y_{1}, y_{2}\right)\right] f_{1}^{\infty}\left(y_{1}\right) f_{2}^{0}\left(y_{2}\right) d y_{1} d y_{2} \mid d z
\end{aligned}
$$

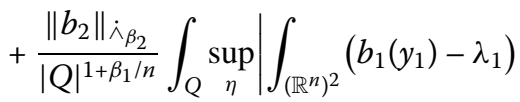

$$
\begin{aligned}
& \times\left[K_{\mu, \eta}\left(z, y_{1}, y_{2}\right)-K_{\mu, \eta}\left(x_{Q}, y_{1}, y_{2}\right)\right] f_{1}^{\infty}\left(y_{1}\right) f_{2}^{\infty}\left(y_{2}\right) d y_{1} d y_{2} \mid d z \\
& \doteq N_{21}+N_{22}+N_{23}+N_{24}+N_{25} \text {. }
\end{aligned}
$$

By the Hölder inequality,

$$
\begin{aligned}
N_{21} & \lesssim\left\|b_{2}\right\|_{{\dot{\beta_{2}}}_{2}}\left(\frac{1}{|Q|^{r^{\prime} \beta_{1} / n+1}} \int_{Q}\left|b_{1}(z)-\lambda_{1}\right|^{r^{\prime}} d z\right)^{1 / r^{\prime}}\left(\frac{1}{|Q|} \int_{Q}\left|U^{*}\left(f_{1}, f_{2}\right)(z)\right|^{r} d z\right)^{1 / r} \\
& \lesssim\left\|b_{1}\right\|_{\dot{\lambda}_{\beta_{1}}}\left\|b_{2}\right\|_{\dot{\lambda}_{\beta_{2}} M_{r}}\left(U^{*}\left(f_{1}, f_{2}\right)\right)(x) .
\end{aligned}
$$

Take $1<q_{1}<p_{1}, 1<q_{2}<p_{2}$, and $1<q<\infty$ such that $1 / q=1 / q_{1}+1 / q_{2}$. Then by the Hölder inequality and by Lemma 3.2,

$$
\begin{aligned}
N_{22} & \lesssim \frac{\left\|b_{2}\right\|_{{\dot{\beta}}_{2}}}{|Q|^{\beta_{1} / n+1 / q}}\left(\int_{Q}\left|U^{*}\left(\left(b_{1}-\lambda_{1}\right) f_{1}^{0}, f_{2}^{0}\right)(z)\right|^{q} d z\right)^{1 / q} \\
& \lesssim \frac{\left\|b_{2}\right\|_{\dot{\lambda}_{\beta_{2}}}}{|Q|^{\beta_{1} / n+1 / q}}\left\|\left(b_{1}-\lambda_{1}\right) f_{1}^{0}\right\|_{L^{q_{1}}}\left\|f_{2}^{0}\right\|_{L^{q_{2}}} \\
& \lesssim\left\|b_{1}\right\|_{{\dot{\beta_{\beta}}}_{\beta_{1}}}\left\|b_{2}\right\|_{{\dot{\beta_{2}}}_{\beta_{2}}} M_{q_{1}}\left(f_{1}\right)(x) M_{q_{2}}\left(f_{2}\right)(x) .
\end{aligned}
$$

For any $y_{2} \in\left(Q^{*}\right)^{c}$, we have $\left|y_{2}-x_{Q}\right| \sim\left|y_{2}-z\right|$ and $\left|z-x_{Q}\right| \leq \frac{\left|y_{2}-z\right|}{2} \leq \frac{1}{2} \max \left\{\left|z-y_{1}\right|\right.$, $\left.\left|z-y_{2}\right|\right\}$. Then by Minkowski's inequality and by Lemma 3.1,

$$
\begin{aligned}
N_{23} \lesssim & \frac{\left\|b_{2}\right\|_{{\dot{\beta_{2}}}_{2}}}{|Q|^{1+\beta_{1} / n}} \int_{Q} \sup _{\eta} \int_{\left(\mathbb{R}^{n}\right)^{2}}\left|\left(b_{1}\left(y_{1}\right)-\lambda_{1}\right)\right| \\
& \times\left|K_{\mu, \eta}\left(z, y_{1}, y_{2}\right)-K_{\mu, \eta}\left(x_{Q}, y_{1}, y_{2}\right)\right|\left|f_{1}^{0}\left(y_{1}\right) f_{2}^{\infty}\left(y_{2}\right)\right| d y_{1} d y_{2} d z \\
\lesssim & \frac{\left\|b_{1}\right\|\left\|_{\lambda_{1}}\right\| b_{2} \|_{\dot{\lambda}_{\beta_{2}}}}{|Q|} \int_{Q} \int_{\left(\mathbb{R}^{n}\right)^{2}} \frac{\left|f_{1}^{0}\left(y_{1}\right) f_{2}^{\infty}\left(y_{2}\right)\right|}{\left(\left|z-y_{1}\right|+\left|z-y_{2}\right|\right)^{2 n}}
\end{aligned}
$$




$$
\begin{aligned}
& \times\left(\omega\left(\frac{\left|z-x_{Q}\right|}{\left|z-y_{1}\right|+\left|z-y_{2}\right|}\right)+\frac{\left|z-x_{Q}\right|}{\left|z-y_{1}\right|+\left|z-y_{2}\right|}\right) d y_{1} d y_{2} d z \\
& \lesssim \frac{\left\|b_{1}\right\|{\dot{\lambda_{\beta_{1}}}}_{1}\left\|b_{2}\right\|_{{\dot{\beta_{3}}}_{2}}}{|Q|} \int_{Q} \int_{Q^{*}}\left|f_{1}\left(y_{1}\right)\right| \int_{\left(Q^{*}\right)^{c}} \frac{\left|f_{2}\left(y_{2}\right)\right|}{\left|z-y_{2}\right|^{2 n}} \\
& \times\left(\omega\left(\frac{\left|z-x_{Q}\right|}{\left|z-y_{2}\right|}\right)+\frac{\left|z-x_{Q}\right|}{\left|z-y_{1}\right|+\left|z-y_{2}\right|}\right) d y_{2} d y_{1} d z \\
& \lesssim \frac{\left\|b_{1}\right\|_{{\dot{\beta_{1}}}_{1}}\left\|b_{2}\right\| \|_{{\dot{\beta_{2}}}_{2}}}{|Q|} \int_{Q} \int_{Q^{*}}\left|f_{1}\left(y_{1}\right)\right| \\
& \times \sum_{k=1}^{\infty} \int_{2^{k+3} \sqrt{n} Q \backslash 2^{k+2} \sqrt{n} Q} \frac{\left(\omega\left(2^{-k}\right)+2^{-k}\right)\left|f_{2}\left(y_{2}\right)\right|}{\left|2^{k} \sqrt{n} Q\right|^{2}} d y_{2} d y_{1} d z \\
& \lesssim\left\|b_{1}\right\|_{{\dot{\beta_{1}}}_{1}}\left\|b_{2}\right\|_{{\dot{\beta}}_{2}} \frac{1}{|Q|} \int_{Q^{*}}\left|f_{1}\left(y_{1}\right)\right| d y_{1} \\
& \times \sum_{k=1}^{\infty} \frac{|Q|}{\left|2^{k+3} \sqrt{n} Q\right|}\left(\omega\left(2^{-k}\right)+2^{-k}\right) \frac{1}{\left|2^{k+3} \sqrt{n} Q\right|} \int_{2^{k+3} \sqrt{n} Q \mid 2^{k+2} \sqrt{n} Q}\left|f_{2}\left(y_{2}\right)\right| d y_{2} \\
& \lesssim C\left\|b_{1}\right\|_{{\dot{\beta_{1}}}_{1}}\left\|b_{2}\right\|_{{\dot{\beta_{2}}}_{2}} M\left(f_{1}\right)(x) M\left(f_{2}\right)(x) \text {. }
\end{aligned}
$$

Similarly, $N_{24} \lesssim\left\|b_{1}\right\|_{{\dot{\beta_{1}}}_{1}}\left\|b_{2}\right\|_{{\dot{\beta_{2}}}_{\beta_{2}}} M\left(f_{1}\right)(x) M\left(f_{2}\right)(x)$.

For any $y_{1}, y_{2} \in\left(Q^{*}\right)^{c}$, we have $\left|y_{1}-x_{Q}\right| \sim\left|y_{1}-z\right|$ and $\left|y_{2}-x_{Q}\right| \sim\left|y_{2}-z\right|$. Then by Minkowski's inequality and by Lemma 3.1 ,

$$
\begin{aligned}
& N_{25} \lesssim \frac{\left\|b_{2}\right\|_{\dot{\lambda}_{\beta_{2}}}}{|Q|^{1+\beta_{1} / n}} \int_{Q} \sup _{\eta} \int_{\left(\mathbb{R}^{n}\right)^{2}}\left|\left(b_{1}\left(y_{1}\right)-\lambda_{1}\right)\right| \\
& \times\left|K_{\mu, \eta}\left(z, y_{1}, y_{2}\right)-K_{\mu, \eta}\left(x_{Q}, y_{1}, y_{2}\right)\right|\left|f_{1}^{\infty}\left(y_{1}\right) f_{2}^{\infty}\left(y_{2}\right)\right| d y_{1} d y_{2} d z \\
& \lesssim \frac{\left\|b_{1}\right\|{\dot{\lambda_{\beta}}}_{\beta_{1}}\left\|b_{2}\right\|{\dot{\lambda_{\beta_{2}}}}_{\mid}}{|Q|^{1+\beta_{1} / n}} \int_{Q} \int_{\left(\mathbb{R}^{n}\right)^{2}} \frac{\left|y_{1}-x_{Q}\right|^{\beta_{1}}\left|f_{1}^{0}\left(y_{1}\right) f_{2}^{\infty}\left(y_{2}\right)\right|}{\left(\left|z-y_{1}\right|+\left|z-y_{2}\right|\right)^{2 n}} \\
& \times\left(\omega\left(\frac{\left|z-x_{Q}\right|}{\left|z-y_{1}\right|+\left|z-y_{2}\right|}\right)+\frac{\left|z-x_{Q}\right|}{\left|z-y_{1}\right|+\left|z-y_{2}\right|}\right) d y_{1} d y_{2} d z \\
& \lesssim \frac{\left\|b_{1}\right\|_{\dot{\lambda}_{\beta_{1}}}\left\|b_{2}\right\|{\dot{\lambda_{\beta_{2}}}}_{\beta^{1+\beta_{1}}}}{|Q|^{1+\beta_{1} / n}} \int_{Q} \int_{\left(\left(Q^{*}\right)^{c}\right)^{2}} \frac{\left|f_{1}\left(y_{1}\right)\right|\left|f_{2}\left(y_{2}\right)\right|}{\left|y_{1}-x_{Q}\right|^{2 n-\beta_{1}}} \\
& \times\left(\omega\left(\frac{\left|z-x_{Q}\right|}{\left|z-y_{1}\right|}\right)+\frac{\left|z-x_{Q}\right|}{\left|z-y_{1}\right|+\left|z-y_{2}\right|}\right) d y_{1} d y_{2} d z
\end{aligned}
$$

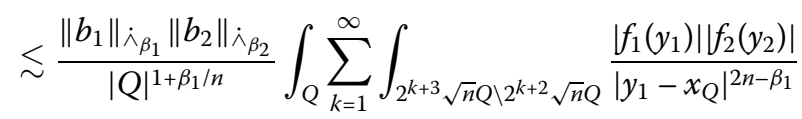

$$
\begin{aligned}
& \times\left(\omega\left(\frac{\left|z-x_{Q}\right|}{\left|z-y_{1}\right|}\right)+\frac{\left|z-x_{Q}\right|}{\left|z-y_{1}\right|+\left|z-y_{2}\right|}\right) d y_{1} d y_{2} d z \\
& \lesssim\left\|b_{1}\right\|{\dot{\lambda_{\beta}}}_{\beta_{1}}\left\|b_{2}\right\|_{\dot{\lambda}_{\beta_{2}}} \sum_{k=1}^{\infty} \frac{2^{k \beta_{1}}\left(\omega\left(2^{-k}\right)+2^{-k}\right)}{\left|2^{k+3} \sqrt{n} Q\right|^{2}} \int_{2^{k+3} \sqrt{n} Q \backslash 2^{k+2} \sqrt{n} Q}\left|f_{1}\left(y_{1}\right)\right| d y_{1} \\
& \times \int_{2^{k+3} \sqrt{n} Q \backslash 2^{k+2} \sqrt{n} Q}\left|f_{2}\left(y_{2}\right)\right| d y_{2} \\
& \lesssim\left\|b_{1}\right\|_{{\dot{\beta_{1}}}_{1}}\left\|b_{2}\right\|_{{\dot{\beta_{2}}}_{2}} M\left(f_{1}\right)(x) M\left(f_{2}\right)(x)
\end{aligned}
$$

where assumption (1.6) was used. 
Combining the estimates for $N_{21}, N_{22}, N_{23}, N_{24}, N_{25}$, we get

$$
N_{2} \lesssim\left\|b_{1}\right\|_{{\dot{\beta_{1}}}_{1}}\left\|b_{2}\right\|_{{\dot{\beta_{2}}}_{2}}\left\{M_{r}\left(U^{*}\left(f_{1}, f_{2}\right)\right)(x)+M_{q_{1}}\left(f_{1}\right)(x) M_{q_{2}}\left(f_{2}\right)(x)+M\left(f_{1}\right)(x) M\left(f_{2}\right)(x)\right\}
$$

The rest of the proof is the same as in [22], and hence we proved Theorem 1.3.

\section{Acknowledgements}

Not applicable.

\section{Funding}

This work was supported partly by the Key Research Project for Higher Education in Henan Province (No. 19A110017) and the National Natural Science Foundation of China (Grant Nos. 11401175, 11571160, and 11471176).

\section{Availability of data and materials}

Not applicable.

\section{Competing interests}

The authors declare that they have no competing interests.

\section{Authors' contributions}

Both authors contributed equally to the manuscript and read and approved the final manuscript.

\section{Author details}

${ }^{1}$ School of Mathematics and Information Science, Henan Polytechnic University, Jiaozuo, People's Republic of China.

${ }^{2}$ Department of Mathematics, Mudanjiang Normal University, Mudanjiang, People's Republic of China.

\section{Publisher's Note}

Springer Nature remains neutral with regard to jurisdictional claims in published maps and institutional affiliations.

Received: 13 February 2019 Accepted: 2 April 2019 Published online: 11 April 2019

\section{References}

1. Anh, B.T., Duong, X.T.: On commutators of vector $B M O$ functions and multilinear singular integrals with non-smooth kernels. J. Math. Anal. Appl. 371, 80-84 (2010)

2. Chen, S., Wu, H.: Multiple weighted estimates for commutators of multilinear singular integrals with non-smooth kernels. J. Math. Anal. Appl. 396, 888-903 (2012)

3. Chen, X.: Weighted estimates for maximal operator of multilinear singular integral. Bull. Pol. Acad. Sci., Math. 58(2) 129-135 (2010)

4. Chen, X., Xue, Q.: Weighted estimates for a class of multilinear fractional type operators. J. Math. Anal. Appl. 362(2), 355-373 (2010)

5. Coifman, R.R., Meyer, Y.: On commutators of singular integrals and bilinear singular integrals. Trans. Am. Math. Soc. 212, 315-331 (1975)

6. Coifman, R.R., Meyer, Y.: Commutateurs d'intégrales singulières et opérateurs multilinéaires. Ann. Inst. Fourier (Grenoble) 28, 177-202 (1978)

7. Coifman, R.R., Meyer, Y.: Au-delà des opérateurs pseudo-différentiels. Asterisque, vol. 57 (1978)

8. Duong, X.T., Mclntosh, A.: Singular integral operators with non-smooth kernels on irregular domains. Rev. Mat. Iberoam. 15, 233-265 (1999)

9. Grafakos, L., He, D., Honzík, P.: Rough bilinear singular integrals. Adv. Math. 326, 54-78 (2018)

10. Grafakos, L., Liu, L., Yang, D.: Multiple weighted norm inequalities for maximal multilinear singular integrals with non-smooth kernels. Proc. R. Soc. Edinb., Sect. A 141, 755-775 (2011)

11. Grafakos, L., Torres, R.H.: Multilinear Calderón-Zygmund theory. Adv. Math. 165, 124-164 (2002)

12. Grafakos, L., Torres, R.H.: Maximal operator and weighted norm inequalities for multilinear singular integrals. Indiana Univ. Math. J. 51(5), 1261-1276 (2002)

13. Hormozi, M., Si, Z., Xue, Q.: On general multilinear square function with non-smooth kernels. Bull. Sci. Math. 149, 1-22 (2018)

14. Lerner, A.K., Ombrosi, S., Pérez, C., Torres, R.H., Trujillo-González, R.: New maximal functions and multiple weights for the multilinear Calderón-Zygmund theory. Adv. Math. 220(4), 1222-1264 (2009)

15. Lu, G., Zhang, P.: Multilinear Calderón-Zygmund operators with kernels of Dini's type and applications. Nonlinear Anal. 107, 92-117 (2014)

16. Mo, H., Lu, S.: Commutators generated by multilinear Calderón-Zygmund type singular integral and Lipschitz functions. Acta Math. Sci. Ser. B Engl. Ed. 40(3), 903-912 (2014)

17. Paluszyński, M.: Characterization of the Besov spaces via the commutator operator of Coifman, Rochberg and Weiss. Indiana Univ. Math. J. 44, 1-18 (1995)

18. Pérez, C., Pradolini, G., Torres, R.H., Trujillo-González, R.: End-point estimates for iterated commutators of multilinear singular integrals. Bull. Lond. Math. Soc. 46(1), 26-42 (2014)

19. Si, Z., Xue, Q.: Weighted estimates for commutators of vector-valued maximal multilinear operators. Nonlinear Anal. 96, 96-108 (2014) 
20. Si, Z., Xue, Q:: A note on vector-valued maximal multilinear operators and their commutators. Math. Inequal. Appl. 19(1), 249-262 (2016)

21. Si, Z., Xue, Q., Yabuta, K.: On the bilinear square Fourier multiplier operators and related square functions. Sci. China Math. 60(8), 1477-1502 (2017)

22. Sun, J., Zhang, P.: Commutators of multilinear Calderón-Zygmund operators with Dini type kernels on some function spaces. J. Nonlinear Sci. Appl. 10(9), 5002-5019 (2017)

23. Wang, W., Xu, J.: Commutators of multilinear singular integrals with Lipschitz functions. Commun. Math. Res. 25(4), 318-328 (2009)

24. Xue, Q.: Weighted estimates for the iterated commutators of multilinear maximal and fractional type operators. Stud. Math. 217(2), 97-122 (2013)

Submit your manuscript to a SpringerOpen ${ }^{\circ}$ journal and benefit from:

- Convenient online submission

- Rigorous peer review

- Open access: articles freely available online

- High visibility within the field

- Retaining the copyright to your article

Submit your next manuscript at $\gg$ springeropen.com 